\title{
Relativeness in quantum gravity: limitations and frame dependence of semiclassical descriptions
}

\author{
Yasunori Nomura, Fabio Sanches and Sean J. Weinberg \\ Berkeley Center for Theoretical Physics, Department of Physics, \\ University of California, Berkeley, CA 94720, U.S.A. \\ Theoretical Physics Group, Lawrence Berkeley National Laboratory, \\ CA 94720, U.S.A. \\ E-mail: ynomura@berkeley.edu, fabios@berkeley.edu, \\ sjweinberg@berkeley .edu
}

ABSTRACT: Consistency between quantum mechanical and general relativistic views of the world is a longstanding problem, which becomes particularly prominent in black hole physics. We develop a coherent picture addressing this issue by studying the quantum mechanics of an evolving black hole. After interpreting the Bekenstein-Hawking entropy as the entropy representing the degrees of freedom that are coarse-grained to obtain a semiclassical description from the microscopic theory of quantum gravity, we discuss the properties these degrees of freedom exhibit when viewed from the semiclassical standpoint. We are led to the conclusion that they show features which we call extreme relativeness and spacetime-matter duality - a nontrivial reference frame dependence of their spacetime distribution and the dual roles they play as the "constituents" of spacetime and as thermal radiation. We describe black hole formation and evaporation processes in distant and infalling reference frames, showing that these two properties allow us to avoid the arguments for firewalls and to make the existence of the black hole interior consistent with unitary evolution in the sense of complementarity. Our analysis provides a concrete answer to how information can be preserved at the quantum level throughout the evolution of a black hole, and gives a basic picture of how general coordinate transformations may work at the level of full quantum gravity beyond the approximation of semiclassical theory.

Keywords: Black Holes in String Theory, Models of Quantum Gravity, Black Holes

ARXIV EPRINT: 1412.7538 


\section{Contents}

1 Introduction 1

2 Failure of global spacetime $\quad 4$

3 Black hole - A distant description 5

3.1 Microscopic structure of a dynamical black hole 6

3.2 Emergence of the semiclassical picture and coarse-graining 9

3.3 "Constituents of spacetime" and their distribution 12

3.4 Hawking emission - "Microscopic" and semiclassical descriptions 15

3.4.1 "Microscopic" (unitary) description 16

$\begin{array}{lll}3.4 .2 & \text { Semiclassical (thermal) description } & 21\end{array}$

3.5 Black hole mining — "Microscopic" and semiclassical descriptions 23

$\begin{array}{ll}3.6 & \text { The fate of an infalling object } \\ \end{array}$

4 Black hole - An infalling description $\quad 27$

4.1 Emergence of interior spacetime - Free fall from a distance 28

4.2 Consistency between the distant and infalling descriptions 31

4.3 Other reference frames - Free fall from a nearby point 34

4.4 (Non-)relations with the Unruh effect in Minkowski space 35

4.5 Complementarity: general covariance in quantum gravity 37

$\begin{array}{lll}5 & \text { Summary - A grand picture } & 37\end{array}$

\section{Introduction}

In the past decades, it has become increasingly apparent that the concept of spacetime must receive substantial revisions when it is treated in a fully quantum mechanical manner. The first clear sign of this came from the study of black hole physics (for a review, see e.g. [1]). Consider describing a process in which an object falls into a black hole, which eventually evaporates, from the viewpoint of a distant observer. Unitarity of quantum mechanics suggests that the information content of the object will first be stored in the black hole system, and then emitted back to distant space in the form of Hawking radiation [2, $3]$. On the other hand, the equivalence principle implies that the object should not find anything special at the horizon, when the process is described by an observer falling with the object. These two pictures lead to inconsistency if we adopt the standard formulation of quantum field theory on curved spacetime, since it allows us to employ a class of equal time hypersurfaces (called nice slices) that pass through both the fallen object and late Hawking radiation, leading to violation of the no-cloning theorem of quantum mechanics [4]. 
In the early 90's, a remarkable suggestion to avoid this difficulty - called complementarity — was made [5]: the apparent cloning of the information occurring in black hole physics implies that the internal spacetime and horizon/Hawking radiation degrees of freedom appearing in different, i.e. infalling and distant, descriptions are not independent. This signals a breakdown of the naive global spacetime picture of general relativity at the quantum level, and it forces us to develop a new view of how classical spacetime arises in the full theory of quantum gravity. One of the main purposes of this paper is to present a coherent picture of this issue. We discuss how a series of well-motivated hypotheses leads to a consistent view of the effective emergence of global spacetime from a fundamental theory of quantum gravity. In particular, we elucidate how this picture avoids the recently raised firewall paradox [6-8], which can be viewed as a refined version of the old information paradox [9]. Our analysis provides a concrete answer to how the information can be preserved at the quantum level in the black hole formation and evaporation processes.

A key element in developing our picture is to identify the origin and nature of the "entropy of spacetime," first discovered by Bekenstein and Hawking in studying black hole physics $[10,11]$. In a previous paper [12], two of us argued that this entropy - the Bekenstein-Hawking entropy - is associated with the degrees of freedom that are coarsegrained to obtain the semiclassical description of the system: quantum theory of matter and radiation on a fixed spacetime background. This picture is consonant with the fact that in quantum mechanics, having a well-defined geometry of spacetime, e.g. a black hole in a well-defined spacetime location, requires taking a superposition of an enormous number of energy-momentum eigenstates, so we expect that there are many different ways to arrive at the same background for the semiclassical theory within the precision allowed by quantum mechanics. This implies that, when a system with a black hole is described in a distant reference frame, the information about the microstate of the black hole is delocalized over a large spatial region, since it is encoded globally in the way of taking the energy-momentum superposition to arrive at the geometry under consideration. In particular, we may naturally identify the spatial distribution of this information as that of the gravitational thermal entropy calculated using the semiclassical theory. This leads to a fascinating picture: the degrees of freedom represented by the Bekenstein-Hawking entropy play dual roles of spacetime and matter - they represent how the semiclassical geometry is obtained at the microscopic level and at the same time can be viewed as the origin of the thermal entropy, which is traditionally associated with thermal radiation in the semiclassical theory.

The delocalization of the microscopic information described above plays an important role in addressing the firewall/information paradox. As described in a distant reference frame, a general black hole state is specified by the following three classes of indices at the microscopic level:

- Indices labeling the (field or string theoretic) degrees of freedom in the exterior spacetime region, excited over the vacuum of the semiclassical theory; ${ }^{1}$

\footnotetext{
${ }^{1}$ Note that the concepts of the breakdown of a semiclassical description and that of semiclassical field theory are not the same - there can be phase space regions in which an object can be well described as a string (or brane) propagating in spacetime, but not as a particle.
} 
- Indices labeling the excitations of the stretched horizon; ${ }^{2}$

- Indices representing the degrees of freedom that are coarse-grained to obtain the semiclassical description, which we will collectively denote by $k$. The information in $k$ represents how the black hole geometry is obtained at the microscopic level, and cannot be resolved by semiclassical operators. It is regarded as being delocalized following the spatial distribution of the gravitational thermal entropy, calculated using the semiclassical theory.

In a distant reference frame, an object falling into the black hole is initially described by the first class of indices, and then by the second when it hits the stretched horizon. The information about the fallen object will then reside there for, at least, time of order $M l_{\mathrm{P}}^{2} \ln \left(M l_{\mathrm{P}}\right)$ (the scrambling time $\left.[13,14]\right)$, after which it will be transmitted to the index $k$. Here, $M$ and $l_{\mathrm{P}}$ are the mass of the black hole and the Planck length, respectively. Finally, the information in $k$, which is delocalized in the whole zone region, will leave the black hole system through the Hawking emission, or black hole mining, process.

Since the microscopic information about the black hole is considered to be delocalized from the semiclassical standpoint, the Hawking emission, or black hole mining, process can be viewed as occurring at a macroscopic distance away from the stretched horizon without contradicting information conservation. In this region, degrees of freedom represented by the index $k$ are converted into modes that have clear identities as semiclassical excitations, i.e. matter or radiation, above the spacetime background. This conversion process, i.e. the emission of Hawking quanta or the excitation of a mining apparatus, is accompanied by the appearance of negative energy excitations, which have negative entropies and propagate inward to the stretched horizon. As we will see, the microscopic dynamics of quantum gravity allows these processes to occur unitarily without violating causality among events described in low energy quantum field theory. This picture avoids firewalls as well as information cloning.

In the description based on a distant reference frame, a falling object can be described by the semiclassical theory only until it hits the stretched horizon, after which it goes outside the applicability domain of the theory. We may, however, describe the fate of the object using the semiclassical language somewhat longer by performing a reference frame change, specifically until the object hits a singularity, after which there is no reference frame that admits a semiclassical description of the object. This reference frame change is the heart of complementarity: the emergence of global spacetime in the classical limit. We argue that while descriptions in different reference frames (the descriptions before and after a complementarity transformation) apparently look very different, e.g. in locations of the degrees of freedom representing the microscopic information of the black hole, their predictions about the same physical question are consistent with each other. This consistency is ensured by an intricate interplay between the properties of microscopic information and the causal structure of spacetime.

\footnotetext{
${ }^{2}$ The stretched horizon is located at a microscopic distance outside of the mathematical horizon, and is regarded as a physical (timelike) membrane which may be physically excited [5].
} 
It is striking that the concept of spacetime, e.g. the region in which a semiclassical description is applicable, depends on a reference frame. This extreme "relativeness" of the description is a result of nonzero Newton's constant $G_{\mathrm{N}}$. The situation is analogous to what happened when the speed of light, $c$, was realized to be finite [15]: in Galilean physics $(c=\infty)$ a change of the reference frame leads only to a constant shift of all the velocities, while in special relativity ( $c=$ finite) it also alters temporal and spatial lengths (time dilation and Lorentz contraction) and makes the concept of simultaneity relative. With gravity $\left(G_{\mathrm{N}} \neq 0\right)$, even the concept of spacetime becomes relative. The trend is consistent - as we "turn on" fundamental constants in nature $\left(c=\infty \rightarrow\right.$ finite and $\left.G_{\mathrm{N}}=0 \rightarrow \neq 0\right)$, physical descriptions become more and more relative: descriptions of the same physical system in different reference frames appear to differ more and more.

The organization of this paper is the following. In section 2, we discuss some basic aspects of the breakdown of global spacetime, setting up the stage for later discussions. In sections 3 and 4 , we describe how our picture addresses the problem of black hole formation and evaporation. We discuss the quantum structure of black hole microstates and the unitary flow of information as viewed from a distant reference frame (in section 3), and how it can be consistent with the existence of interior spacetime (in section 4). In particular, we elucidate how this picture addresses the arguments for firewalls and provides a consistent resolution to the black hole information paradox. In section 5, we give our summary by presenting a grand picture of the structure of quantum gravity implied by our analysis of a system with a black hole.

Throughout the paper, we adopt the Schrödinger picture for quantum evolution, and use natural units in which $\hbar=c=1$ unless otherwise stated. We limit our discussions to 4-dimensional spacetime, although we do not expect difficulty in extending to other dimensions. The value of the Planck length in our universe is $l_{\mathrm{P}}=G_{\mathrm{N}}^{1 / 2} \simeq 1.62 \times 10^{-35} \mathrm{~m}$. A concise summary of the implications of our framework for black hole physics can be found in ref. [16].

\section{Failure of global spacetime}

As described in the introduction, semiclassical theory applied to an entire global spacetime leads to overcounting of the true degrees of freedom at the quantum level. This implies that in the full theory of quantum gravity, a semiclassical description of physics emerges only in some limited sense. Here we discuss basic aspects of this limitation, setting up the stage for later discussions.

The idea of complementarity [5] is that the overcounting inherent in the global spacetime picture may be avoided if we limit our description to what a single "observer" represented by a single worldline in spacetime - can causally access. Depending on which observer we choose, we obtain different descriptions of the system, which are supposed to be equivalent. Since the events an observer can see lie within the causal patch associated with the worldline representing the observer, we may assume that this causal patch is the spacetime region a single such description may represent. In particular, one may postulate the following $[15,17]$ : 
- For a single description allowing a semiclassical interpretation of the system, the spacetime region represented is restricted to the causal patch associated with a single worldline. With this restriction, the description can be local in the sense that any physical correlations between low energy field theoretic degrees of freedom respect causality in spacetime (beyond some microscopic quantum gravitational distance $l_{*}$, meaning that possible nonlocal corrections are exponentially suppressed $\sim e^{-r / l_{*}}$ ).

Depending on the worldline we take, we may obtain different descriptions of the same system, which are all local in appropriate spacetime regions. A transformation between different descriptions is nothing but the complementarity transformation.

To implement Hamiltonian quantum mechanics, we must introduce a time variable. This corresponds to foliating the causal patch by equal-time hypersurfaces, with a state vector $|\Psi(t)\rangle$ representing the state of the system on each hypersurface. ${ }^{3}$ Let $\mathbf{x}$ be spatial coordinates parameterizing each equal-time hypersurface. Physical quantities associated with field theoretic degrees of freedom can then be obtained using field theoretic operators $\phi(\mathbf{x})$ and the state $|\Psi(t)\rangle$. (Excited string degrees of freedom will require the corresponding operators.) In general, the procedure of electing coordinates $(t, \mathbf{x})$, which we need to define states and operators, must be given independently of the background spacetime, since we do not know it a priori (and states may even represent superpositions of very different semiclassical geometries); an example of such procedures is described in ref. [20]. In our discussions in this paper, however, we mostly consider issues addressed on a fixed background spacetime (at least approximately), so we need not be concerned with this problem too much - we may simply use any coordinate system adapted to a particular spacetime we consider, e.g. Schwarzschild-like coordinates for a black hole.

In the next two sections, we discuss how the complementarity picture described above works for a dynamical black hole. We discuss the semiclassical descriptions of the system in various reference frames, as well as their mutual consistency. In these discussions, we focus on a black hole that is well approximated by a Schwarzschild black hole in asymptotically flat spacetime. We do not expect difficulty in extending it to more general cases.

\section{Black hole - A distant description}

Suppose we describe the formation and evaporation of a black hole in a distant reference frame. Following refs. [2, 3], we postulate that there exists a unitary description which involves only the degrees of freedom that can be viewed as being on and outside the (stretched) horizon. To describe quantum states with a black hole, we adopt Schwarzschildlike time slicings to define equal-time hypersurfaces. ${ }^{4}$ We argue that the origin of the

\footnotetext{
${ }^{3}$ In general, the "time variable" of (constrained) Hamiltonian quantum mechanics may not be related directly with time we observe in nature [18]. Indeed, the whole "multiverse" may be represented by a state that does not depend on the time variable and is normalizable in an appropriate sense [19]. Even if this is the case, however, when we describe only a branch of the whole state, e.g. when we describe a system seen by a particular observer, the state of the system may depend on time. In this paper, we discuss systems with black holes, which are parts of the multiverse so their states may depend on time.

${ }^{4}$ Strictly speaking, to describe a general gravitating system we need a procedure to foliate the relevant spacetime region in a background independent manner, as discussed in the previous section. For our present
} 
Bekenstein-Hawking entropy may be viewed as a coarse-graining performed to obtain a semiclassical description of the evolving black hole. We then discuss implications of such a coarse-graining, in particular how it reconciles unitarity of the Hawking emission and black hole mining processes in the fundamental theory with the non-unitary (thermal) view in the semiclassical description.

\subsection{Microscopic structure of a dynamical black hole}

Consider a quantum state which represents a black hole of mass $M$ located at some place at rest, where the position and velocity are measured with respect to some distant reference frame, e.g. an inertial frame elected at asymptotic infinity. Because of the uncertainty principle, such a state must involve a superposition of energy and momentum eigenstates. Let us first estimate the required size of the spread of energy $\Delta E$, with $E$ measured in the asymptotic region. According to the standard Hawking calculation, a state of a black hole of mass $M$ will evolve after Schwarzschild time $\Delta t \approx O\left(M l_{\mathrm{P}}^{2}\right)$ into a state representing a Hawking quantum of energy $\approx O\left(1 / M l_{\mathrm{P}}^{2}\right)$ and a black hole with the correspondingly smaller mass. The fact that these two states - before and after the emission - are nearly orthogonal implies that the original state must involve a superposition of energy eigenstates with

$$
\Delta E \approx \frac{1}{\Delta t} \approx O\left(\frac{1}{M l_{\mathrm{P}}^{2}}\right)
$$

Of course, this is nothing but the standard time-energy uncertainty relation, and here we have assumed that a state after time $t \ll M l_{\mathrm{P}}^{2}$ is not clearly distinguishable from the original one, so that the uncertainty relation is almost saturated.

Next, we consider the spread of momentum $\Delta p$, where $p$ is again measured in the asymptotic region. Suppose we want to identify the spatial location of the black hole with precision comparable to the quantum stretching of the horizon $\Delta r \approx O(1 / M)$, i.e. $\Delta d \approx O\left(l_{\mathrm{P}}\right)$, where $r$ and $d$ are the Schwarzschild radial coordinate and the proper length, respectively. This implies that the superposition must involve momenta with spread $\Delta p \approx$ $\left(1 / M l_{\mathrm{P}}\right)(1 / \Delta d) \approx O\left(1 / M l_{\mathrm{P}}^{2}\right)$, where the factor $1 / M l_{\mathrm{P}}$ in the middle expression is the redshift factor. This value of $\Delta p$ corresponds to an uncertainty of the kinetic energy $\Delta E_{\text {kin }} \approx p \Delta p / M \approx O\left(1 / M^{3} l_{\mathrm{P}}^{4}\right)$, which is much smaller than $\Delta E$ in eq. (3.1). The spread of energy thus comes mostly from a superposition of different rest masses: $\Delta E \approx \Delta M$.

How many different independent ways are there to superpose the energy eigenstates to arrive at the same black hole geometry, at a fixed position within the precision specified by $\Delta r$ and of mass $M$ within an uncertainty of $\Delta M$ ? We assume that the Bekenstein-Hawking

purposes, however, it suffices to employ any foliation that reduces to Schwarzschild-like time slicings when the black hole exists. Note that macroscopic uncertainties in the black hole mass, location, and spin caused by the stochastic nature of Hawking radiation [21, 22] require us to focus on appropriate branches in the full quantum state in which the black hole in a given time has well-defined values for these quantities at the classical level. The relation between the Schwarzschild-like foliation and a general background independent foliation is then given by the standard coordinate transformation, which does not introduce subtleties beyond those discussed in this paper. The effect on unitarity by focusing on particular branches in this way is also minor, so we ignore it. The full unitarity, however, can be recovered by keeping all the branches in which the black hole has different classical properties at late times [22]. 
entropy, $\mathcal{A} / 4 l_{\mathrm{P}}^{2}$, gives the logarithm of this number (at the leading order in expansion in inverse powers of $\mathcal{A} / l_{\mathrm{P}}^{2}$ ), where $\mathcal{A}=16 \pi M^{2} l_{\mathrm{P}}^{4}$ is the area of the horizon. While the definition of the Bekenstein-Hawking entropy does not depend on the precise values of $\Delta M$ or $\Delta p$, a natural choice for these quantities is

$$
\Delta M \approx \Delta p \approx O\left(\frac{1}{M l_{\mathrm{P}}^{2}}\right),
$$

which we will adopt. The nonzero Bekenstein-Hawking entropy thus implies that there are exponentially many independent states in a small energy interval of $\Delta E \approx O\left(1 / M l_{\mathrm{P}}^{2}\right)$. We stress that it is not appropriate to interpret this to mean that quantum mechanics introduces exponentially large degeneracies that do not exist in classical black holes. In classical general relativity, a set of Schwarzschild black holes located at some place at rest are parameterized by a continuous mass parameter $M$; i.e., there are a continuously infinite number of black hole states in the energy interval between $M$ and $M+\Delta M$ for any $M$ and small $\Delta M$. Quantum mechanics reduces this to a finite number $\approx e^{S_{0}} \Delta M / M$, with $S_{0}$ given $\mathrm{by}^{5}$

$$
S_{0}=\frac{\mathcal{A}}{4 l_{\mathrm{P}}^{2}}+O\left(\frac{\mathcal{A}^{q}}{l_{\mathrm{P}}^{2 q}} ; q<1\right) .
$$

This can also be seen from the fact that $S_{0}$ is written as $\mathcal{A} c^{3} / 4 l_{\mathrm{P}}^{2} \hbar$ when $\hbar$ and $c$ are restored, which becomes infinite for $\hbar \rightarrow 0$.

As is clear from the argument above, there are exponentially many independent microstates, corresponding to eq. (3.3), which are all black hole vacuum states: the states that do not have a field or string theoretic excitation on the semiclassical black hole background and in which the stretched horizon, located at $r_{\mathrm{s}}=2 M l_{\mathrm{P}}^{2}+O(1 / M)$, is not excited. ${ }^{6}$ Denoting the indices representing these exponentially many states collectively by $k$, which we call the vacuum index, basis states for the general microstates of a black hole of mass $M$ (within the uncertainty of $\Delta M$ ) can be given by

$$
\left|\Psi_{\bar{a} a a_{\text {far }} ; k}(M)\right\rangle \text {. }
$$

Here, $\bar{a}, a$, and $a_{\text {far }}$ represent the indices labeling the excitations of the stretched horizon, in the near exterior zone region (i.e. the region within the gravitational potential barrier defined, e.g., as $\left.r \leq R_{\mathrm{Z}} \equiv 3 M l_{\mathrm{P}}^{2}\right)$, and outside the zone $\left(r>R_{\mathrm{Z}}\right)$, respectively. ${ }^{7}$ As we

\footnotetext{
${ }^{5}$ Of course, quantum mechanics allows for a superposition of these finite number of independent states, so the number of possible (not necessarily independent) states is continuously infinite. The statement here applies to the number of independent states, regarding classical black holes with different $M$ as independent states.

${ }^{6}$ These states can be defined, for example, as the states obtained by first forming a black hole of mass $M$ and then waiting sufficiently long time after (artificially) switching off Hawking emission. Note that at the level of full quantum gravity, all the black hole states are obtained as excited states. Any semiclassical description, however, treats some of them as vacuum states on the black hole background.

${ }^{7}$ Strictly speaking, the states may also have the vacuum index associated with the ambient space in which the black hole exists. The information in this index, however, is not extracted in the Hawking evaporation or black hole mining process, so we ignore it here. (For more discussions, see, e.g., section 5 of ref. [12].) We will also treat excitations spreading both in the $r \leq R_{\mathrm{Z}}$ and $r>R_{\mathrm{Z}}$ regions only approximately by including them either in $a$ or $a_{\mathrm{far}}$. The precise description of these excitations will require more elaborate expressions, e.g. than the one in eq. (3.6), which we believe is an inessential technical subtlety in addressing our problem.
} 
have argued, the index $k$ runs over $1, \cdots, e^{S_{0}}$ for the vacuum states $\bar{a}=a=a_{\text {far }}=0$. In general, the range for $k$ may depend on $\bar{a}$ and $a$, but its dependence is higher order in $l_{\mathrm{P}}^{2} / \mathcal{A}$; i.e., for fixed $\bar{a}$ and $a$

$$
k=1, \cdots, e^{S_{\bar{a} a}} ; \quad S_{\bar{a} a}-S_{0} \approx O\left(\frac{\mathcal{A}^{q}}{l_{\mathrm{P}}^{2 q}} ; q<1\right)
$$

We thus mostly ignore this small dependence of the range of $k$ on $(\bar{a}, a)$, i.e. the nonfactorizable nature of the Hilbert space factors spanned by these indices, except when we discuss negative energy excitations associated with Hawking emission later, where this aspect plays a relevant role in addressing one of the firewall arguments.

Since we are mostly interested in physics associated with the black hole region, we also introduce the notation in which the excitations in the far exterior region are separated. As we will see later, the degrees of freedom represented by $k$ can be regarded as being mostly in the region $r \leq R_{Z}$, so we may write the states of the entire system in eq. (3.4) as

$$
\left|\Psi_{\bar{a} a a_{\mathrm{far}} ; k}(M)\right\rangle \approx\left|\psi_{\bar{a} a ; k}(M)\right\rangle\left|\phi_{a_{\mathrm{far}}}(M)\right\rangle,
$$

and call $\left|\psi_{\bar{a} a ; k}(M)\right\rangle$ and $\left|\phi_{a_{\mathrm{far}}}(M)\right\rangle$ as the black hole and exterior states, respectively. Note that by labeling the states in terms of localized excitations, we need not write explicitly the trivial vacuum entanglement between the black hole and exterior states that does not depend on $k$, which typically exist when they are specified in terms of the occupation numbers of modes spanning the entire space.

How many independent quantum states can the black hole region support? Let us label appropriately coarse-grained excitations in the region $r_{\mathrm{s}} \leq r \leq R_{\mathrm{Z}}$ by $i=1,2, \cdots$, each of which carries entropy $S_{i}$. Suppose there are $n_{i}$ excitations of type $i$ at some fixed locations. The entropy of such a configuration is given by the sum of the "entropy of vacuum" in eq. (3.3) and the entropies associated with the excitations:

$$
S_{I}=S_{0}+\sum_{i} n_{i} S_{i}
$$

The energy of the system in the region $r \leq R_{\mathrm{Z}}$ is given by the sum of the mass $M$ of the black hole, which we define as the energy the system would have in the absence of an excitation outside the stretched horizon, and the energies associated with the excitations in the zone. Note that excitations here are defined as fluctuations with respect to a fixed background, so their energies $E_{i}$ as well as entropies $S_{i}$ can be either positive or negative, although the signs of the energy and entropy must be the same: $E_{i} S_{i}>0$. The meaning of negative entropies will be discussed in detail in sections 3.4 and 3.5.

Since excitations in the zone affect geometry, spacetime outside the stretched horizon, when they exist, is not exactly that of a Schwarzschild black hole. We require that these excitations do not form a black hole by themselves or become a part of the black hole at the center; otherwise, the state must be viewed as being built on a different semiclassical vacuum. ${ }^{8}$ The total entropy $S$ of the region $r \leq R_{\mathrm{Z}}$, i.e. the number of independent

\footnotetext{
${ }^{8}$ More precisely, we regard two geometries as being built on different classes of semiclassical vacua when they have different horizon configurations as viewed from a fixed reference frame. On the other hand, if two
} 
microscopic quantum states representing this region, is then given by

$$
S=\ln \left(\sum_{I} e^{S_{I}}\right)
$$

where $I$ represents possible configurations of excitations, specified by the set of numbers $\left\{n_{i}\right\}$ and the locations of excitations of each type $i$, that do not modify the semiclassical vacuum in the sense described above. As suggested by a representative estimate [23], and particularly emphasized in ref. [24], the contribution of such excitations to the total entropy is subdominant in the expansion in inverse powers of $\mathcal{A} / l_{\mathrm{P}}^{2}: S=S_{0}+O\left(\mathcal{A}^{q} / l_{\mathrm{P}}^{2 q} ; q<1\right)$. The total entropy in the near black hole region, $r \leq R_{\mathrm{Z}}$, is thus given by

$$
S=\frac{\mathcal{A}}{4 l_{\mathrm{P}}^{2}}
$$

at the leading order in $l_{\mathrm{P}}^{2} / \mathcal{A}$.

\subsection{Emergence of the semiclassical picture and coarse-graining}

The fact that all the independent microstates with different values of $k$ lead to the same geometry suggests that the semiclassical picture is obtained after coarse-graining the degrees of freedom represented by this index; namely, any result in semiclassical theory is a statement about the maximally mixed ensemble of microscopic quantum states consistent with the specified background within the precision allowed by quantum mechanics [12]. According to this picture, the black hole vacuum state in the semiclassical description is given by the density matrix

$$
\rho_{0}(M)=\frac{1}{e^{S_{0}}} \sum_{k=1}^{e^{S_{0}}}\left|\Psi_{\bar{a}=a=a_{\mathrm{far}}=0 ; k}(M)\right\rangle\left\langle\Psi_{\bar{a}=a=a_{\mathrm{far}}=0 ; k}(M)\right| .
$$

Because of the coarse-graining of an enormous number of degrees of freedom, this density matrix has statistical characteristics.

In order to obtain the response of this state to the operators in the semiclassical theory, we may trace out the subsystem on which they do not act. As we will discuss more later, the operators in the semiclassical theory in general act on a part, but not all, of the degrees of freedom represented by the $k$ index. Let us denote the subsystem on which semiclassical operators act nontrivially by $C$, and its complement by $\bar{C}$. The index $k$ may then be viewed as labeling the states in the combined $C \bar{C}$ system which satisfy certain constraints, e.g. the total energy being $M$ within $\Delta M$. The density matrix representing the semiclassical vacuum state in the Hilbert space in which the semiclassical operators act nontrivially, $C$, is given by

$$
\tilde{\rho}_{0}(M)=\operatorname{Tr}_{\bar{C}} \rho_{0}(M)
$$

geometries have the same horizon, they belong to the same "vacuum equivalence class" in the sense that one can be converted into the other with "excitations." For more discussions on this point, see ref. [20] and section 3.2 . 
Consistently with our identification of the origin of the Bekenstein-Hawking entropy, we assume that this density matrix represents the thermal density matrix with temperature $T_{\mathrm{H}}=1 / 8 \pi M l_{\mathrm{P}}^{2}$ in the zone region (as measured at asymptotic infinity):

$$
\tilde{\rho}_{0}(M) \approx \frac{1}{\operatorname{Tr} e^{-\beta H_{\mathrm{sc}}(M)}} e^{-\beta H_{\mathrm{sc}}(M)} ; \quad \beta=\left\{\begin{array}{l}
\frac{1}{T_{\mathrm{H}}} \text { for } r \leq R_{\mathrm{Z}}, \\
+\infty \text { for } r>R_{\mathrm{Z}}
\end{array}\right.
$$

where $H_{\mathrm{sc}}(M)$ is the Hamiltonian of the semiclassical theory in the distant reference frame, which is defined in the region $r \geq r_{\mathrm{s}}$ on the black hole background of mass $M .^{9}$ (The meaning of position-dependent $\beta$ is that the expression $\beta H_{\mathrm{sc}}(M)$ should be interpreted as $\beta$ times the Hamiltonian density integrated over space.) Note that this procedure of obtaining eq. (3.12) from eq. (3.10) can be viewed as an example of the standard procedure of obtaining the canonical ensemble of a system from the microcanonical ensemble of a larger (isolated) system that contains the system of interest. In fact, if the system traced out is larger than the system of interest, $\operatorname{dim} \bar{C} \gtrsim \operatorname{dim} C$, we expect to obtain the canonical ensemble in this manner (see ref. [25] for a related discussion). Below, we drop the tilde from the density matrix in eq. (3.12), as it represents the same state as the one in eq. (3.10) $\rho_{0}(M)$ must be interpreted to mean either the right-hand side of eq. (3.10) or of eq. (3.12), depending on the Hilbert space under consideration.

In semiclassical field theory, the density matrix of eq. (3.12) is obtained as a reduced density matrix by tracing out the region within the horizon in the unique global black hole vacuum state. Our view is that this density matrix, in fact, is obtained from a mixed state of exponentially many pure states, arising from a coarse-graining performed in eq. (3.10); the prescription in the semiclassical theory provides (merely) a useful way of obtaining the same density matrix, in a similar sense in which the thermofield double state was originally introduced [26]. We emphasize that the information in $k$ is invisible in the semiclassical theory (despite the fact that it involves subsystem $C$ ) as it is already coarse-grained to obtain the theory; in particular, the dynamics of the degrees of freedom represented by $k$ cannot be described in terms of the semiclassical Hamiltonian $H_{\mathrm{sc}}(M) .{ }^{10}$ As we will see explicitly later, it is this inaccessibility of $k$ that leads to the apparent violation of unitarity in the semiclassical calculation of the Hawking emission process [9]. Note that because $\rho_{0}(M)$ takes the form of the maximally mixed state in $k$, results in the semiclassical theory do not depend on the basis of the microscopic states chosen in this space.

\footnotetext{
${ }^{9}$ The Hilbert space of the semiclassical theory for states which have a single black hole at a fixed location at rest may be decomposed as $\mathcal{H}=\oplus_{M} \mathcal{H}_{M}$, where $\mathcal{H}_{M}$ is the space spanned by the states in which there is a black hole of (appropriately coarse-grained) mass $M$. In this language, $H_{\mathrm{sc}}(M)$ is a part of the semiclassical Hamiltonian acting on the subspace $\mathcal{H}_{M}$.

${ }^{10}$ This does not mean that a device made out of semiclassical degrees of freedom cannot probe information in $k$. Since there are processes in the fundamental theory (i.e. Hawking evaporation and mining processes) in which information in $k$ is transferred to that in semiclassical excitations (i.e. degrees of freedom represented by the $a$ and $a_{\text {far }}$ indices), information in $k$ can be probed by degrees of freedom appearing in the semiclassical theory. It is simply that these information extraction processes cannot be described within the semiclassical theory, since it can make statements only about the ensemble in eq. (3.10) and excitations built on it.
} 
A comment is in order. In connecting the expression in eq. (3.10) to eq. (3.12), we have (implicitly) assumed that $\left|\Psi_{\bar{a}=a=a_{\mathrm{far}}=0 ; k}(M)\right\rangle$ represent the black hole vacuum states in the limit that the effect from evaporation is (artificially) shut off. ${ }^{11}$ With this definition of vacuum states, the evolution effect necessarily "excites" the states, making $a \neq 0$, as we will see more explicitly in section 3.4. As a consequence, the density matrix for the semiclassical operators representing the evolving black hole deviates from eq. (3.12) even without matter or radiation. (In the semiclassical picture, this is due to the fact that the effective gravitational potential is not truly confining, so that the state of the black hole is not completely stationary.) If one wants, one can redefine vacuum states to be these states: the states that do not have any matter or radiation excitation on the evolving black hole background - the original vacuum states are then obtained as excited states on the new vacuum states. ${ }^{12}$ This redefinition is possible because the two semiclassical "vacua" represented by the two classes of microstates belong to the same "vacuum equivalence class" in the sense described in the last paragraph of section 3.1; specifically, they possess the same horizon for the same black hole mass, as defined for the evaporating case in refs. $[29,30]$.

As was mentioned above, semiclassical operators, in particular those for modes in the zone, act nontrivially on both $a$ and $k$ indices of microstates $\left|\Psi_{\bar{a} a a_{\mathrm{far}} ; k}(M)\right\rangle$. This can be seen as follows. If the operators acted only on the $a$ index, the maximal mixture in $k$ space with $a=0$, eq. (3.10), would look like a pure state from the point of view of these operators, contradicting the thermal nature in eq. (3.12). On the other hand, if the operators acted only on the $k$ index, they would commute with the maximally mixed state in $k$ space, again contradicting the thermal state. Since the thermal nature of eq. (3.12) is prominent only for modes whose energies as measured in the asymptotic region are of order the Hawking temperature or smaller

$$
\omega \lesssim T_{\mathrm{H}}
$$

i.e. whose energies as measured by local (approximately) static observers are of order or smaller than the blueshifted Hawking temperature $T_{\mathrm{H}} / \sqrt{1-2 M l_{\mathrm{P}}^{2} / r}$, this feature is significant only for such infrared modes - operators representing modes with $\omega \gg T_{\mathrm{H}}$ act essentially only on the $a$ index. For operators representing the modes with eq. (3.13), their actions on microstates can be very complicated, although they act on the coarse-grained vacuum state of eq. (3.10) as if it is the thermal state in eq. (3.12), up to corrections suppressed by the exponential of the vacuum entropy $S_{0}$. The commutation relations of these operators defined on the coarse-grained states take the form as in the semiclassical theory, again up to exponentially suppressed corrections.

\footnotetext{
${ }^{11}$ This is analogous to the treatment of a meta-stable vacuum in usual quantum field theory. At the most fundamental level (or on a very long timescale), such a state must be viewed as a scattering state built on the true ground state of the system. In practice (or on a sufficiently short timescale), however, we regard it as a vacuum state, which is approximately the ground state of a theory in which the tunneling out of this state is artificially switched off, e.g. by making the relevant potential barriers infinitely high.

${ }^{12}$ In the standard language in semiclassical theory, the original vacuum states correspond essentially to the Hartle-Hawking vacuum [27], while the new ones (very roughly) to the Unruh vacuum [28].
} 
There is a simple physical picture for this phenomenon of "non-decoupling" of the $a$ and $k$ indices for the infrared modes. As viewed from a distant reference frame, these modes are "too soft" to be resolved clearly above the background - since the derivation of the semiclassical theory involves coarse-graining over microstates in which the energy stored in the region $r \lesssim R_{\mathrm{Z}}$ has spreads of order $\Delta E \approx 1 / M l_{\mathrm{P}}^{2}$, infrared modes with $\omega \lesssim T_{\mathrm{H}} \approx O\left(1 / M l_{\mathrm{P}}^{2}\right)$ are not necessarily distinguished from "spacetime fluctuations" of order $\Delta E$. One might think that if a mode has nonzero angular momentum or charge, one can discriminate it from spacetime fluctuations. In this case, however, it cannot be clearly distinguished from vacuum fluctuations of a Kerr or Reissner-Nordström black hole having the corresponding (minuscule) angular momentum or charge. In fact, we may reverse the logic and view that this lack of a clear identity of the soft modes is the physical origin of the thermality of black holes (and thus of Hawking radiation).

Once the state for the vacuum of the semiclassical theory is obtained as in eq. (3.10) (or eq. (3.12) after partial tracing) and appropriate coarse-grained operators acting on it are identified, it is straightforward to construct the rest of the states in the theory we simply have to act these operators (either field theoretic or of excited string states) on $\rho_{0}(M)$ to obtain the excited states. For example, to obtain a state which has a field theoretic excitation in the zone, one can apply the appropriate linear combination of creation and/or annihilation operators in the semiclassical theory, $a_{\omega \ell m}^{\dagger}$ and/or $a_{\omega \ell m}$ :

$$
\begin{aligned}
\rho_{\bar{a}=0 \text { a } a_{\mathrm{far}}=0}(M)= & \left(\sum_{\ell, m} \int\left(c_{\omega \ell m}^{a} a_{\omega \ell m}+c_{\omega \ell m}^{\prime a} a_{\omega \ell m}^{\dagger}\right) d \omega\right) \\
& \times \rho_{0}(M)\left(\sum_{\ell, m} \int\left(c_{\omega \ell m}^{a} a_{\omega \ell m}+c_{\omega \ell m}^{\prime a} a_{\omega \ell m}^{\dagger}\right) d \omega\right)^{\dagger},
\end{aligned}
$$

where $c_{\omega \ell m}^{a}$ and $c_{\omega \ell m}^{\prime a}$ are coefficients. In the case that the applied operator is that for an infrared mode, this represents a state in which the thermal distribution for the infrared modes is "modulated" by an excitation over it. A construction similar to eq. (3.14) also works for excitations in the far region. To obtain excitations of the stretched horizon, i.e. $\bar{a} \neq 0$, operators dedicated to describing them must be introduced. The detailed dynamics of these degrees of freedom, i.e. the $r=r_{\mathrm{s}}$ part of $H_{\mathrm{sc}}(M)$, is not yet fully known, however.

\section{3 "Constituents of spacetime" and their distribution}

While not visible in semiclassical theory, the black hole formation and evaporation (or mining) processes do involve the degrees of freedom represented by $k$, which we call finegrained vacuum degrees of freedom, or vacuum degrees of freedom for short. The dynamics of these degrees of freedom as well as their interactions with the excitations in the semiclassical theory are determined by the fundamental theory of quantum gravity, which is not yet well known. We may, however, anticipate their basic properties based on some general considerations. In particular, motivated by the general idea of complementarity, we assume the following: 
- Interactions with vacuum degrees of freedom do not introduce violation of causality among field theory degrees of freedom (except possibly for exponentially suppressed corrections, $\sim e^{-r / l_{*}}$ with $l_{*}$ a short-distance quantum gravitational scale).

- Interactions between vacuum degrees of freedom and excitations in the semiclassical theory are such that unitarity is preserved at the microscopic level.

The first assumption is a special case of the postulate discussed in section 2, applied to the distant reference frame description of a black hole. This implies that we cannot send superluminal signals among field theory degrees of freedom using interactions with vacuum degrees of freedom. The second assumption has an implication for how the vacuum degrees of freedom may appear from the semiclassical standpoint, which we now discuss.

In quantum mechanics, the information about a state is generally delocalized in space - locality is a property of dynamics, not that of states. In the case of black hole states, the information about $k$, which roughly represents slightly different "values" (superpositions) of $M$, is generally delocalized in a large spatial region, so that it can be accessed physically in a region away from the stretched horizon (e.g. around the edge of the zone $r \sim R_{\mathrm{Z}}$ ). This, however, does not mean that the complete information about the state can be recovered by a physical process occurring in a limited region in spacetime. For example, if we consider the set of $e^{S_{0}}$ different black hole vacuum states, a physical detector occupying a finite spatial region can only partially discriminate these states in a given finite time.

To see how much information a physical detector in spatial region $i$ can resolve, we can consider the reduced density matrix obtained after tracing out the subsystems that cannot be accessed by the semiclassical degrees of freedom associated with this region. In particular, we may consider the set of all field theory (and excited string state) operators that have support in $i$, and trace out the subsystems that do not respond to any of these operators (which we denote by $\bar{C}_{i}$ ):

$$
\rho_{0}^{(i)}=\operatorname{Tr}_{\bar{C}_{i}} \rho_{0}(M),
$$

where $\rho_{0}(M)$ is given by eq. (3.10), and we have omitted the argument $M$ for $\rho_{0}^{(i)}$. The von Neumann entropy of this density matrix, $S_{0}^{(i)}=-\operatorname{Tr} \rho_{0}^{(i)} \ln \rho_{0}^{(i)}$, then indicates the discriminatory power the region $i$ possesses - a physical process occurring in region $i$ can, at most, discriminate the $e^{S_{0}}$ states into $e^{S_{0}^{(i)}}\left(\ll e^{S_{0}}\right)$ types in a characteristic timescale of the system, $1 / \Delta E \approx O\left(M l_{\mathrm{P}}^{2}\right)$. According to the assumption in eq. (3.12), this entropy is the gravitational thermal entropy contained in region $i$, calculated using the semiclassical theory.

We therefore arrive at the following picture. Let us divide the region $r \geq r_{\mathrm{s}}$ into $N$ (arbitrary) subregions, each of which is assumed to have a sufficiently large number of degrees of freedom so that the thermodynamic limit can be applied. A basis state in the semiclassical theory can be written as

$$
\rho_{\bar{a} a a_{\mathrm{far}}}(M)=\rho_{a_{1}}^{(1)} \otimes \rho_{a_{2}}^{(2)} \otimes \cdots \otimes \rho_{a_{N}}^{(N)},
$$

where $\rho_{a_{i}}^{(i)}$ are states defined in the $i$-th subregion, with $a_{i}$ representing excitations contained in that region. (Following the convention in section 3.2, we regard the vacuum states, 
$\bar{a}=a=a_{\mathrm{far}}=0$, to be defined in the limit that the effect from evaporation is ignored.) Now, in the full Hilbert space of quantum gravity, there are $e^{S_{0}}$ independent states that all reduce to the same $\rho_{\bar{a} a a_{\text {far }}}(M)$ at the semiclassical level. These states can be written as

$$
\left|\Psi_{\bar{a} a a_{\mathrm{far}} ; k=\left\{k_{i}\right\}}(M)\right\rangle=\left|\psi_{a_{1} ; k_{1}}^{(1)}\right\rangle\left|\psi_{a_{2} ; k_{2}}^{(2)}\right\rangle \cdots\left|\psi_{a_{N} ; k_{N}}^{(N)}\right\rangle,
$$

where $k_{i}=1, \cdots, e^{S_{0}^{(i)}}$ with

$$
S_{0}^{(i)} \approx \text { gravitational thermal entropy contained in subregion } i,
$$

calculated using the semiclassical theory for subregions that do not contain the stretched horizon. The $S_{0}^{(i)}$ 's for the subregions involving the stretched horizon are determined by the condition

$$
\sum_{i=1}^{N} S_{0}^{(i)}=S_{0} \approx \frac{\mathcal{A}}{4 l_{\mathrm{P}}^{2}},
$$

which is valid in the thermodynamic limit. Assuming that the entropy on the stretched horizon is distributed uniformly on the surface, this condition determines the entropies contained in all the subregions.

The association of $k_{i}$ 's to each subregion, as in eq. (3.17), corresponds to taking a specific basis in the space spanned by $k$. While the expressions above are strictly valid only in the thermodynamic limit, the corrections caused by deviating from it (e.g. due to correlations among subregions) do not affect our later discussions. In particular, it does not change the fact that the region around the edge of the zone, $r \leq R_{\mathrm{Z}}$ and $r-2 M l_{\mathrm{P}}^{2} \nless M l_{\mathrm{P}}^{2}$, contains $O(1)$ bits of information about $k$ (as it contains $O(1)$ bits of gravitational thermal entropy), which becomes important when we discuss the Hawking emission process in section 3.4. Incidentally, the picture described here leads to the natural interpretation that the subsystem that is traced out when going from eq. (3.10) to eq. (3.12) corresponds to the stretched horizon; i.e. $\bar{C}$ lives on the stretched horizon, while $C$ in the zone. ${ }^{13}$

We stress that by the gravitational thermal entropy in eq. (3.18), we mean that associated with the equilibrium vacuum state. It counts the thermal entropy within the zone, since this region is regarded as being in equilibrium because of its boundedness due to the stretched horizon and the potential barrier; on the other hand, eq. (3.18) does not count the thermal entropy associated with Hawking radiation emitted from the zone, which is (artificially) switched off in defining our vacuum microstates. In other words, when calculating $S_{0}^{(i)}$ 's using eq. (3.18) we should use the vacuum state in eq. (3.12), implying that we should use the local temperature, i.e. the temperature as measured by local static

\footnotetext{
${ }^{13}$ This in turn gives us a natural prescription to determine the location of the stretched horizon precisely. Since the semiclassical expression in eq. (3.12) is expected to break down for $\ln \operatorname{dim} C>\ln \operatorname{dim} \bar{C}$, a natural place to locate the stretched horizon, i.e. the cutoff of the semiclassical spacetime, is where the gravitational thermal entropy outside the stretched horizon becomes $S_{0} / 2=\mathcal{A} / 8 l_{\mathrm{P}}^{2}$. For $n$ low energy species, this yields $r_{\mathrm{s}}-2 M l_{\mathrm{P}}^{2} \sim n / M \sim l_{*}^{2} / M l_{\mathrm{P}}^{2}$, where $l_{*}$ is the string (cutoff) scale and we have used the relation $l_{*}^{2} \sim n l_{\mathrm{P}}^{2}$, which is expected to apply in any consistent theory of quantum gravity (see, e.g., ref. [31]). This scaling is indeed consistent, giving the local Hawking temperature at the stretched horizon $T\left(r_{\mathrm{s}}\right) \sim 1 / l_{*}$, where $T(r)$ is given in eq. (3.20).
} 
observers, of

$$
T(r) \simeq \begin{cases}\frac{T_{\mathrm{H}}}{\sqrt{1-\frac{2 M l_{\mathrm{P}}^{2}}{r}}} & \text { for } r \leq R_{\mathrm{Z}}, \\ 0 & \text { for } r>R_{\mathrm{Z}} .\end{cases}
$$

When the evolution effect is turned on, which we will analyze in section 3.4, the state of the zone is modified $(a \neq 0)$ due to an ingoing negative energy flux, while the state outside the zone is excited $\left(a_{\mathrm{far}} \neq 0\right)$ by Hawking quanta, which are emitted from the edge of the zone and propagate freely in the ambient space. The contribution of the negative energy flux to the entropy within the zone is small, as we will see in section 3.4.

The distribution of vacuum degrees of freedom in eqs. (3.17), (3.18) is exactly the one needed for the interactions between these degrees of freedom and semiclassical excitations to preserve unitarity [12]. Imagine we put a physical detector at constant $r$ in the zone. The detector then sees the thermal bath for all the modes with blueshifted Hawking temperature, eq. (3.20), including higher angular momentum modes. This allows for the detector(s) to extract energy from the black hole at an accelerated rate compared with spontaneous Hawking emission: the mining process [32, 33]. In order for this process to preserve unitarity, the detector must also extract information at the correspondingly accelerated rate. This is possible if the information about the microstate of the black hole, specified by the index $k$, is distributed according to the gravitational thermal entropy, as in eqs. (3.17), (3.18). A similar argument also applies to the spontaneous Hawking emission process, which is viewed as occurring around the edge of the zone, $r \sim R_{Z}$, where the gravitational thermal entropy is small but not negligible. The microscopic and semiclassical descriptions of these processes will be discussed in detail in sections 3.4 and 3.5.

It is natural to interpret the expression in eq. (3.17) to mean that $k_{i}$ labels possible configurations of "physical soft quanta" — or the "constituents of spacetime" — that comprise the region $i$. In a certain sense, this interpretation is correct. The dimension of the relevant Hilbert space, $e^{S_{0}^{(i)}}$, controls possible interactions of the vacuum degrees of freedom with the excitations in the semiclassical theory in region $i$, e.g. how much information a detector located in region $i$ can extract from the vacuum degrees of freedom. This simple picture, however, breaks down when we describe the same system from a different reference frame. As we will discuss in section 4 , the distribution of the vacuum degrees of freedom depends on the reference frame - they are not "anchored" to spacetime. Nevertheless, in a fixed reference frame, the concept of the spatial distribution of the degrees of freedom represented by the index $k$ does make sense. In particular, in a distant reference frame the distribution is given by the gravitational thermal entropy calculated in the semiclassical theory, as we discussed here.

\subsection{Hawking emission - "Microscopic" and semiclassical descriptions}

The formation and evaporation of a black hole involve processes in which the information about the initial collapsing matter is transferred into the vacuum index $k$, which will later be transferred back to the excitations in the semiclassical theory, i.e. the state of final 
Hawking radiation. Schematically, we may write these processes as

$$
\left|m_{\text {init }}\right\rangle \rightarrow \sum_{k=1}^{e^{S_{0}(M(t))}} \sum_{l} c_{k l}(t)\left|\psi_{k}(M(t))\right\rangle\left|r_{l}(t)\right\rangle \rightarrow\left|r_{\text {fin }}\right\rangle,
$$

where $\left|m_{\text {init }}\right\rangle,\left|\psi_{k}(M(t))\right\rangle,\left|r_{l}(t)\right\rangle$, and $\left|r_{\text {fin }}\right\rangle$ represent the states for the initial collapsing matter, the black hole of mass $M(t)$ (which includes the near exterior zone region; see eq. (3.6)), the subsystem complement to the black hole at time $t$, and the final Hawking quanta after the black hole is completely evaporated, respectively. Here, we have suppressed the indices representing excitations for the black hole states. For generic initial states and microscopic emission dynamics, this evolution satisfies the behavior outlined in ref. [34] on general grounds.

In this subsection, we discuss how the black hole evaporating process in eq. (3.21) proceeds in details, elucidating how the arguments for firewalls in refs. [6-8] are avoided. We also discuss how the semiclassical theory describes the same process, elucidating how the thermality of Hawking radiation arises despite the unitarity of the process at the fundamental level.

\subsection{1 "Microscopic" (unitary) description}

Let us first consider how the "elementary" Hawking emission process is described at the microscopic level, ${ }^{14}$ i.e. how a "single" Hawking emission occurs in the absence of any excitations other than those directly associated with the emission. (As we will see later, this is not a very good approximation in general, but the treatment here is sufficient to illustrate the basic mechanism by which the information is transferred from the black hole to the ambient space.)

Suppose a black hole of mass $M$ is in microstate $k$ :

$$
\left|\Psi_{k}(M)\right\rangle=\left|\psi_{k}(M)\right\rangle\left|\phi_{I}\right\rangle
$$

where $\left|\psi_{k}(M)\right\rangle$ is the black hole state, in which we have omitted indices representing excitations, while $\left|\phi_{I}\right\rangle$ is the exterior state, from which we have suppressed small $M$ dependence (which, e.g., causes a small gravitational redshift of a factor of about 1.5 for the emitted Hawking quanta to reach the asymptotic region). As discussed in sections 3.2 and 3.3, we consider $\left|\Psi_{k}(M)\right\rangle$ to be one of the black hole vacuum microstates in the limit that the evolution effect is shut off; see, e.g., eqs. (3.12), (3.20). The effect of the evolution, which consists of successive elementary Hawking emission processes, will be discussed later.

After a timescale of $t \approx O\left(M l_{\mathrm{P}}^{2}\right)$, the state in eq. (3.22) evolves due to Hawking emission as

$$
\left|\psi_{k}(M)\right\rangle\left|\phi_{I}\right\rangle \rightarrow \sum_{i, a, k^{\prime}} c_{i a k^{\prime}}^{k}\left|\psi_{a ; k^{\prime}}(M)\right\rangle\left|\phi_{I+i}\right\rangle
$$

\footnotetext{
${ }^{14}$ By the "microscopic" description, we mean a description in which the vacuum index $k$ is kept (i.e. not coarse-grained as in the semiclassical description) so that the process is manifestly unitary at each stage of the evolution. A complete description of the microscopic dynamics of the vacuum degrees of freedom requires the fundamental theory of quantum gravity, which is beyond the scope of this paper.
} 
where $\left|\phi_{I+i}\right\rangle$ is the state in which newly emitted Hawking quanta, labeled by $i$ and having total energy $E_{i}$, are added to the appropriately time evolved $\left|\phi_{I}\right\rangle$. The index $a$ represents the fact that the black hole state has negative energy excitations of total energy $-E_{a}$ $\left(E_{a}>0\right)$ around the edge of the zone, created in connection with the emitted Hawking quanta; the coefficients $c_{i a k^{\prime}}^{k}$ are nonzero only if $E_{i} \approx E_{a}$ (within the uncertainty). ${ }^{15}$ The negative energy excitations then propagate inward, and after a time of order $M l_{\mathrm{P}}^{2} \ln \left(M l_{\mathrm{P}}\right)$ collide with the stretched horizon, making the black hole states relax as

$$
\left|\psi_{a ; k^{\prime}}(M)\right\rangle \rightarrow \sum_{k_{a}} d_{k_{a}}^{a k^{\prime}}\left|\psi_{k_{a}}\left(M-E_{a}\right)\right\rangle
$$

The combination of eqs. (3.23), (3.24) yields

$$
\left|\psi_{k}(M)\right\rangle\left|\phi_{I}\right\rangle \rightarrow \sum_{i, k_{i}} \alpha_{i k_{i}}^{k}\left|\psi_{k_{i}}\left(M-E_{i}\right)\right\rangle\left|\phi_{I+i}\right\rangle,
$$

where $\alpha_{i k_{i}}^{k}=\sum_{a, k^{\prime}} c_{i a k^{\prime}}^{k} d_{k_{i}}^{a k^{\prime}}$, and we have used $E_{i}=E_{a}$; here, $M-E_{i}$ for different $i$ may belong to the same mass within the precision $\Delta M$, i.e. $M-E_{i}=M-E_{i^{\prime}}$ for $i \neq i^{\prime}$. This expression shows that information in the black hole can be transferred to the radiation state $i$.

It is important that the negative energy excitations generated in eq. (3.23) come with negative entropies, so that each of the processes in eqs. (3.23), (3.24) (as well as the propagation of the negative energy excitations in the zone) is separately unitary. This means that as $k$ and $i$ run over all the possible values with $a$ being fixed, the index $k^{\prime}$ runs only over $1, \cdots, e^{S_{0}\left(M-E_{a}\right)}$, the dimension of the space spanned by $k_{a}$. In fact, this is an example of the non-factorizable nature of the Hilbert space factors spanned by $k$ and $a$ discussed in eq. (3.5), which we assume to arise from the fundamental theory. This structure of the Hilbert space allows for avoiding the argument for firewalls in ref. [7] — unlike what is imagined there, elements of the naive Fock space built on each $k$ in a way isomorphic to that of quantum field theory are not all physical; the physical Hilbert space is smaller than such a (hypothetical) Fock space. This implies, in particular, that the Fock space structure of a semiclassical theory does not factor from the space spanned by the vacuum index $k$, as is also implied by the analysis in section 3.2.

To further elucidate the point made above, we can consider the following simplified version of the relevant processes. Suppose a black hole in a superposition state of $\left|\psi_{k}(M)\right\rangle$ 's $\left(k=1, \cdots, e^{S_{0}(M)}\right)$ releases 1 bit of information through Hawking emission of the form:

$$
\left|\psi_{k}(M)\right\rangle\left|\phi_{0}\right\rangle \rightarrow\left\{\begin{array}{l}
\left|\psi_{a ; \frac{k+1}{2}}(M)\right\rangle\left|\phi_{1}\right\rangle \text { if } k \text { is odd, } \\
\left|\psi_{a ; \frac{k}{2}}(M)\right\rangle\left|\phi_{2}\right\rangle \text { if } k \text { is even, }
\end{array}\right.
$$

where we have assumed $E_{1}=E_{2}=(\ln 2) / 8 \pi M l_{\mathrm{P}}^{2} \simeq T_{\mathrm{H}}$, so that the entropy of the black hole after the emission is reduced by 1 bit: $S_{0}\left(M-E_{1}\right)=S_{0}(M)-\ln 2$. Note that the

\footnotetext{
${ }^{15}$ To be precise, the sum in the right-hand side of eq. (3.23) contains the " $i=0$ terms" representing the branches in which no quantum is emitted: $\left|\phi_{I+0}\right\rangle=\left|\phi_{I}\right\rangle$. In these terms, there is no negative energy excitation: $c_{0 a k^{\prime}}^{k} \neq 0$ only for $a=0$. The following expressions are valid including these terms with the definition $E_{i=0}=E_{a=0}=0$.
} 
index representing the negative energy excitation (of energy $-E_{1}$ ) takes the same value $a$ in the first and second lines. Namely, while the entire process in eq. (3.26) is unitary, the initial states with $k=2 n-1$ and $2 n$ lead to the same black hole state. After the negative energy excitation reaches the stretched horizon, the black hole states relax into vacuum states for a smaller black hole:

$$
\left|\psi_{a ; k^{\prime}}(M)\right\rangle \rightarrow\left|\psi_{k_{1}=k^{\prime}}\left(M-E_{1}\right)\right\rangle .
$$

While the resulting black hole has a smaller entropy than the original black hole, this relaxation process is unitary because $k^{\prime}$ in the left-hand side runs only over $1, \cdots, e^{S_{0}(M)} / 2=$ $e^{S_{0}\left(M-E_{1}\right)}$. We note that the creation of a positive energy Hawking quantum and a negative energy excitation in eq. (3.26) (and in eq. (3.23)) takes a form very different from the standard "pair creation" of particles, which is often invoked to visualize the Hawking emission process. In the pair creation picture, the positive and negative energy excitations are maximally entangled with each other, which is not the case here. In fact, it is this lack of entanglement that allows the emission process to transfer the information from the black hole to radiation.

We emphasize that from the semiclassical spacetime viewpoint, the emission of eq. (3.23) is viewed as occurring locally around the edge of the zone, which is possible because the information about the black hole microstate extends into the whole zone region according to eqs. (3.17), (3.18). To elucidate this point, we may consider the tortoise coordinate

$$
r^{*}=r+2 M l_{\mathrm{P}}^{2} \ln \frac{r-2 M l_{\mathrm{P}}^{2}}{2 M l_{\mathrm{P}}^{2}},
$$

in which the region outside the Schwarzschild horizon $r \in\left(2 M l_{\mathrm{P}}^{2}, \infty\right)$ is mapped into $r^{*} \in(-\infty, \infty)$. This coordinate is useful in that the kinetic term of an appropriately redefined field takes the canonical form, so that its propagation can be analyzed as in flat space. In this coordinate, the stretched horizon, located at $r=2 M l_{\mathrm{P}}^{2}+O\left(l_{*}^{2} / M l_{\mathrm{P}}^{2}\right)($ see footnote 13), is at

$$
r_{\mathrm{s}}^{*} \simeq-4 M l_{\mathrm{P}}^{2} \ln \frac{M l_{\mathrm{P}}^{2}}{l_{*}} \simeq-4 M l_{\mathrm{P}}^{2} \ln \left(M l_{\mathrm{P}}\right),
$$

where $l_{*}$ is the string (or gravitational cutoff) scale, which we take to be within a couple of orders of magnitude of $l_{\mathrm{P}}$. This implies that there is a large distance between the stretched horizon and the potential barrier region when measured in $r^{*}: \Delta r^{*} \approx 4 M l_{\mathrm{P}}^{2} \ln \left(M l_{\mathrm{P}}\right) \gg$ $O\left(M l_{\mathrm{P}}^{2}\right)$ for $\ln \left(M l_{\mathrm{P}}\right) \gg 1$. On the other hand, a localized Hawking quantum is represented by a wavepacket with width of $O\left(M l_{\mathrm{P}}^{2}\right)$ in $r^{*}$, since it has an energy of order $T_{\mathrm{H}}=1 / 8 \pi M l_{\mathrm{P}}^{2}$ defined in the asymptotic region.

The point is that, given the state $\left|\Psi_{k}(M)\right\rangle=\left|\psi_{k}(M)\right\rangle\left|\phi_{I}\right\rangle$, the process in eq. (3.23) occurs in the region $\left|r^{*}\right| \approx O\left(M l_{\mathrm{P}}^{2}\right)$ (i.e. the region in which the effective gravitational potential starts shutting off toward large $r^{*}$ ) without involving deep interior of the zone $-r^{*} \gg M l_{\mathrm{P}}^{2}$. In this region, information stored in the vacuum state is converted into that of a particle state outside the zone. More specifically, the information in the vacuum represented by the $k$ index (which may also be viewed as a thermal bath of infrared 


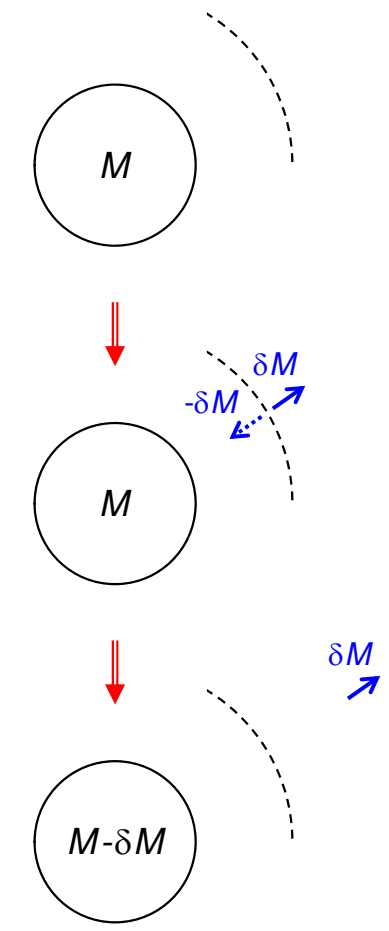

Figure 1. A schematic picture of the elementary Hawking emission process; time flows from the top to the bottom. The edge of the zone, i.e. the barrier region of the effective gravitational potential, is shown by a portion of a dashed circle at each moment in time. The emitted Hawking quanta as well as negative energy excitations are depicted by arrows (solid and dotted, respectively) although they are mostly $s$-waves.

modes, eq. (3.13), though only in certain senses) is transferred into that in modes $a_{\mathrm{far}} \neq 0$, i.e. Hawking quanta, which have clear independent identities over the background spacetime. Due to energy conservation, this process is accompanied by the creation of ingoing negative energy excitations; however, they are not maximally entangled with the emitted Hawking quanta.

In figure 1, we depict schematically the elementary Hawking emission process described here. In the figure, we have denoted the emitted Hawking quanta as well as negative energy excitations by arrows, although they are mostly $s$-waves [35]. The discussion here makes it clear that the purifiers of the emitted Hawking quanta in the Hawking emission process are microstates which semiclassical theory describes as a vacuum. In particular, the emission process does not involve any excitation which, in the near horizon Rindler approximation, appears as a mode breaking entanglement between the two Rindler wedges necessary to keep the horizon smooth. Outgoing Hawking quanta emerge at the edge of the zone, living outside the applicability of the Rindler approximation. Ingoing negative energy excitations appear, in the Rindler approximation, as modes smooth in Minkowski space, which involve necessary entanglements between Rindler modes in the two wedges and have frequencies of order $1 / M l_{\mathrm{P}}^{2}$ in the Minkowski frame. Unlike what was considered in ref. [6], and unlike what a "naive" interpretation of semiclassical theory might seem to suggest, 
Hawking quanta are not modes associated solely with one of the Rindler wedges ( $b$ modes in the notation of ref. [6]) nor outgoing Minkowski modes ( $a$ modes), which would appear to have high energies for observers who are freely falling into the black hole. This allows for avoiding the entropy argument for firewalls given in ref. [6] as well as the typicality argument in ref. [8].

In the discussion of the Hawking emission so far, we have assumed that a single emission of Hawking quanta as well as the associated creation of ingoing negative energy excitations occur in a black hole vacuum state consisting of $\left|\Psi_{k}(M)\right\rangle$ 's, which are defined in the limit that the evolution effect is ignored. In reality, however, there are always of order $\ln \left(M l_{\mathrm{P}}\right)$ much of negative energy excitations in the zone, since the emission process occurs in every time interval of order $M l_{\mathrm{P}}^{2}$ and the time it takes for a negative energy excitation to reach the stretched horizon is of order $M l_{\mathrm{P}}^{2} \ln \left(M l_{\mathrm{P}}\right.$ ) (both measured in the asymptotic region) - an evaporating black hole has an ingoing flux of negative energy excitations of entropy $\approx O\left(-\ln \left(M l_{\mathrm{P}}\right)\right)$ at all times. This flux of excitations modifies spacetime geometry from that of a Schwarzschild black hole; in particular, the geometry near the horizon is well described by the advanced/ingoing Vaidya metric [29, 30]. Note that as discussed in section 3.2, we may redefine our vacuum states to include these negative energy excitations, although we do not do it here.

Finally, it is instructive to consider the time reversal of the Hawking emission process. In this case, radiation coming from the far exterior region and outgoing negative energy excitations emitted from the stretched horizon meet around the edge of the zone; see figure 2(a). This results in a black hole state of mass given by the sum of the mass $M$ of the original black hole (before emitting the negative energy excitations) and the energy $\delta M$ of the incoming radiation. It is a "vacuum" state in the sense that there is no excitation in the zone except for those associated with a steady flux of outgoing negative energy excitations. We emphasize that this process is very different from what happens when generic incoming radiation of energy $\delta M \approx O\left(1 / M l_{\mathrm{P}}^{2}\right)$ is sent to a usual (i.e. evaporating, not anti-evaporating) black hole. In this case, the radiation enters into the zone without being "annihilated" by a negative energy excitation, which after hitting the stretched horizon will lead to a black hole state of mass $M+\delta M$; see figure 2(b). In fact, the process in figure 2(a) is a process which leads to a decrease of coarse-grained (or thermal) entropy, as implied by the fact that the coarse-grained entropy increases in the standard Hawking emission process [36, 37]. In order for this to happen, therefore, the initial radiation and black hole state must be exponentially fine-tuned; otherwise, the radiation would simply propagate inward in the zone as depicted in figure 2(b) (although it can be subject to significant scattering by the effective gravitational potential at the time of the entrance). The origin of the conversion from radiation to vacuum degrees of freedom for such a fine-tuned initial state can be traced to the non-decoupling of the $a$ and $k$ indices discussed in section 3.2. ${ }^{16}$

\footnotetext{
${ }^{16}$ If the black hole vacuum states are redefined as discussed in section 3.2, the outgoing negative energy flux cannot be seen as excitations. The physics described here, however, will not change; in particular, only exponentially fine-tuned initial states allow for converting radiation to vacuum degrees of freedom around the edge of the zone.
} 

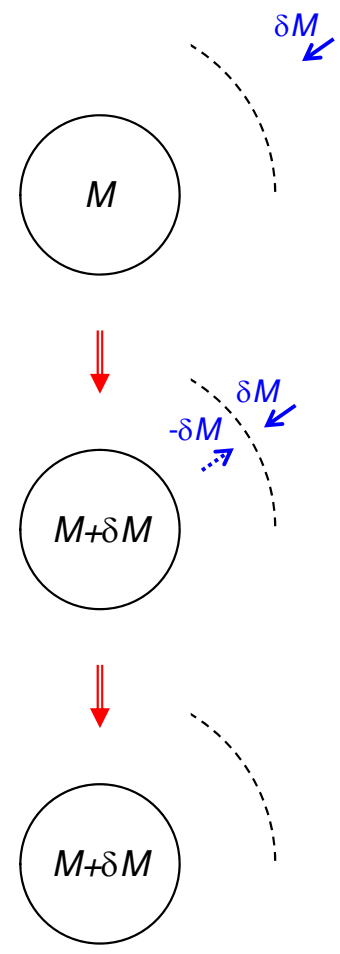

(a) Time reversal of figure 1
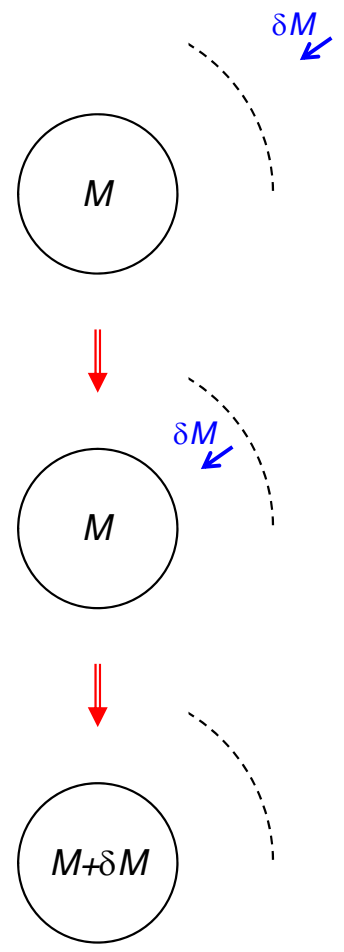

(b) Generic incoming radiation

Figure 2. Time reversal of the Hawking emission process (a) as opposed to the process in which generic incoming radiation enters into the zone of a usual black hole (b). The former is an entropy decreasing process requiring an exponentially fine-tuned initial state, while the latter is a standard process respecting the (generalized) second law of thermodynamics.

\subsubsection{Semiclassical (thermal) description}

The expression in eq. (3.21) implies that at an intermediate stage of the evolution, the information about the initial collapsing matter is encoded in the black hole microstates labeled by $k$ and their entanglement with the rest of the system (which will later be transformed into the state of final-state Hawking radiation). Since semiclassical theory is incapable of describing the dynamics associated with the index $k$, it leads to apparent violation of unitarity at all stages of the black hole formation and evaporation processes. In particular, the state of the emitted Hawking quanta in each time interval of order $M(t) l_{\mathrm{P}}^{2}$ is given by the incoherent thermal superposition with temperature $1 / 8 \pi M(t) l_{\mathrm{P}}^{2}$, making the final Hawking radiation state a mixed thermal state - this is an intrinsic limitation of the semiclassical description, which involves a coarse-graining.

To see in detail how thermal Hawking radiation in the semiclassical picture results from unitary evolution at the fundamental level, let us analyze the elementary Hawking emission process given in eq. (3.25). Following eq. (3.10), we consider the "semiclassical vacuum state" with a black hole of mass $M$, obtained after taking the maximally mixed 
ensemble of microstates:

$$
\rho(M)=\frac{1}{e^{S_{0}(M)}} \sum_{k=1}^{e^{S_{0}(M)}}\left|\psi_{k}(M)\right\rangle\left|\phi_{I}\right\rangle\left\langle\psi_{k}(M)\right|\left\langle\phi_{I}\right| .
$$

The evolution of this state under eq. (3.25) is then given by

$$
\begin{aligned}
\rho(M) \rightarrow \frac{1}{e^{S_{0}(M)}} & \sum_{k=1}^{e^{S_{0}(M)}} \sum_{i, i^{\prime}} \\
& \sum_{k_{i}=1}^{e_{0}\left(M-E_{i}\right)} \sum_{k_{i^{\prime}}=1}^{S_{0}\left(M-E_{i^{\prime}}\right)} \alpha_{i k_{i}}^{k} \alpha_{i^{\prime} k_{i^{\prime}}^{\prime}}^{k *}\left|\psi_{k_{i}}\left(M-E_{i}\right)\right\rangle\left|\phi_{I+i}\right\rangle\left\langle\psi_{k_{i^{\prime}}^{\prime}}\left(M-E_{i^{\prime}}\right)\right|\left\langle\phi_{I+i^{\prime}}\right| .
\end{aligned}
$$

Now, assuming that the microscopic dynamics of the vacuum degrees of freedom are generic, we expect using $S_{0}(M)=4 \pi M^{2} l_{\mathrm{P}}^{2}$ that tracing out the black hole states leads to

$\operatorname{Tr}\left[\frac{1}{e^{S_{0}(M)}} \sum_{k=1}^{e^{S_{0}(M)}} \sum_{k_{i}=1}^{e^{S_{0}\left(M-E_{i}\right)}} \sum_{k_{i^{\prime}}^{\prime}=1}^{e^{S_{0}\left(M-E_{i^{\prime}}\right)}} \alpha_{i k_{i}}^{k} \alpha_{i^{\prime} k_{i^{\prime}}^{\prime}}^{k *}\left|\psi_{k_{i}}\left(M-E_{i}\right)\right\rangle\left\langle\psi_{k_{i^{\prime}}^{\prime}}\left(M-E_{i^{\prime}}\right)\right] \approx \frac{1}{Z} g_{i} e^{-\frac{E_{i}}{T_{\mathrm{H}}}} \delta_{i i^{\prime}}\right.$,

where $T_{\mathrm{H}}=1 / 8 \pi M l_{\mathrm{P}}^{2}, Z=\sum_{i} g_{i} e^{-E_{i} / T_{\mathrm{H}}}$, and $g_{i}$ is a factor that depends on $i$. This allows us to write the reduced density matrix representing the exterior state after the evolution in eq. (3.31) as

$$
\rho_{\mathrm{ext}} \approx \frac{1}{Z} \sum_{i} g_{i} e^{-\frac{E_{i}}{T_{\mathrm{H}}}}\left|\phi_{I+i}\right\rangle\left\langle\phi_{I+i}\right|,
$$

which is the result obtained in Hawking's original calculation, with $g_{i}$ representing the gray-body factor calculable in the semiclassical theory [35].

The analysis given above elucidates why the semiclassical calculation sees apparent violation of unitarity in the Hawking emission process, i.e. why the final expression in eq. (3.33) does not depend on microstates of the black hole, despite the fact that the elementary process in eq. (3.25) is unitary, so that the coefficients $\alpha_{i k_{i}}^{k}$ depend on $k$. It is because the semiclassical calculation (secretly) deals with the mixed state, eq. (3.30), from the beginning - states in semiclassical theory are maximal mixtures of black hole microstates labeled by vacuum indices, i.e. $k$ 's. By construction, the semiclassical theory cannot capture unitarity of detailed microscopic processes involving these indices, including the black hole formation and evaporation processes.

We finally discuss how the unitarity and thermal nature of the black hole evaporation process may appear in (thought) experiments, illuminating physical implications of the picture described here. Suppose we prepare an ensemble of a large number of black holes of mass $M$ all of which are in an identical microstate $k$, and collect the Hawking quanta emitted from these black holes in a time interval of order $M l_{\mathrm{P}}^{2}$. The quanta emitted from each black hole are then in the same quantum state throughout the ensemble, so that a measurement of the spectrum of all the emitted quanta does not reveal the thermal property predicted by the semiclassical theory. On the other hand, if the members of the ensemble 
are in different microstates distributed randomly in $k$ space, then the collection of the Hawking quanta emitted from all the black holes do exhibit the thermal nature consistent with the prediction of the semiclassical theory within the Hilbert space describing the quanta emitted from each black hole (which has dimension only of order unity).

What is the significance of the thermal nature for a single black hole, rather than an ensemble of a large number of black holes? If we form a black hole of mass $M$ in a particular microstate $k$ and collect all the Hawking quanta emitted throughout the evaporation process without measuring them along the way, then the state of the quanta contains the complete information about $k$, reflecting unitarity of the process at the fundamental level - the concept of thermality does not apply to this particular state as a whole. On the other hand, if an observer measures Hawking quanta emitted in each time interval of order $M(t) l_{\mathrm{P}}^{2}$, then the (incoherent) ensemble of measurement outcomes does exhibit the thermal nature as predicted by the semiclassical theory. ${ }^{17}$ Since this is the kind of measurement that a realistic observer typically makes, the semiclassical theory can be said to provide a good prediction even for the outcome of (a series of) measurements a single observer performs on a single black hole.

\subsection{Black hole mining - "Microscopic" and semiclassical descriptions}

It is known that one can accelerate the energy loss rate of a black hole faster than that of spontaneous Hawking emission by extracting its energy from the thermal atmosphere using a physical apparatus: the mining process. This acceleration occurs largely because the number of "channels" one can access increases by going into the zone - unlike the case of spontaneous Hawking emission, which is dominated by $s$-wave radiation, higher angular momentum modes can also contribute to the energy loss in this process [33]. Note that the rate of energy loss associated with each channel, however, is still the same order as that in the spontaneous Hawking emission process: energy of order $E \approx O\left(1 / M l_{\mathrm{P}}^{2}\right)$ is lost in each time interval of $t \approx O\left(M l_{\mathrm{P}}^{2}\right)$, with $E$ and $t$ both defined in the asymptotic region. This fact will become important in section 4 when we discuss the mining process as viewed from an infalling reference frame.

The information transfer associated with the mining process occurs in a similar way to that in the spontaneous Hawking emission process. An essential difference is that since the process involves higher angular momentum modes, the negative energy excitations arising from backreactions can now be localized in angular directions. Specifically, consider a physical detector (or a system of detectors) located at a fixed Schwarzschild radial coordinate $r=r_{\mathrm{d}}$ within the zone, $r_{\mathrm{s}}<r_{\mathrm{d}}<R_{\mathrm{Z}}$. The detector then responds as if it is immersed in the thermal bath of blueshifted Hawking temperature $T\left(r_{\mathrm{d}}\right)$, with $T(r)$ given by eq. (3.20).

\footnotetext{
${ }^{17}$ In the more fundamental, many-world picture, this implies that the record of a physical observer who has "measured," or interacted with, emitted quanta in multiple moments shows a result consistent with the thermality predicted by the semiclassical theory. Note that a single branch in which such an observer lives does not in general contain the whole information about the initial black hole state $k$. The complete information about $k$ (as well as that of the initial state of the observer) is contained only in a state given by a superposition of all possible branches resulting from interactions (and non-interactions) between the observer and quanta, representing all the possible "outcomes" the observer could have had (the probability distribution of which is consistent with thermality).
} 
Suppose the detector has the ground state $\left|d_{0}\right\rangle$ and excited states $\left|d_{i}\right\rangle(i=1,2, \ldots)$ playing the role of the "ready" state and pointer states, respectively, and that the proper energies needed to excite $\left|d_{0}\right\rangle$ to $\left|d_{i}\right\rangle$ are given by $E_{\mathrm{d}, i}$. The mining process can then be written such that after a timescale of $t \approx O\left(M l_{\mathrm{P}}^{2}\right.$ ) (as measured in the asymptotic region), the state of the combined black hole and detector system evolves as

$$
\left|\psi_{k}(M)\right\rangle\left|d_{0}\right\rangle \rightarrow \sum_{i, a, k^{\prime}} c_{i a k^{\prime}}^{k}\left|\psi_{a ; k^{\prime}}(M)\right\rangle\left|d_{i}\right\rangle,
$$

where we have assumed, as in the discussion of "elementary" Hawking emission, that there are no excitations other than those directly associated with the process. The state $\left|\psi_{a ; k^{\prime}}(M)\right\rangle$ arises as a result of backreaction of the detector response; it contains a negative energy excitation $a$ with energy $-E_{a}$, which is generally localized in angular directions. The coefficients $c_{i a k^{\prime}}^{k}$ are nonzero only if $E_{a} \approx E_{\mathrm{d}, i} \sqrt{1-2 M l_{\mathrm{P}}^{2} / r_{\mathrm{d}}}$ within the uncertainty.

Once created, the negative energy excitations propagate inward, and after time of $t \approx r_{\mathrm{d}}^{*}-r_{\mathrm{s}}^{*}$ collide with the stretched horizon, where $r^{*}$ is the tortoise coordinate in eq. (3.28). This will make the black hole states relax as

$$
\left|\psi_{a ; k^{\prime}}(M)\right\rangle \rightarrow \sum_{k_{a}} d_{k_{a}}^{a k^{\prime}}\left|\psi_{k_{a}}\left(M-E_{a}\right)\right\rangle
$$

in the scrambling time of $t \approx O\left(M l_{\mathrm{P}}^{2} \ln \left(M l_{\mathrm{P}}\right)\right)$. As in the case of spontaneous Hawking emission, this relaxation process is unitary because the negative energy excitations carry negative entropies; i.e. for a fixed $a$, the index $k^{\prime}$ runs only over $1, \cdots, e^{S_{0}\left(M-E_{a}\right)} \ll e^{S_{0}(M)}$. The combination of eqs. (3.34), (3.35) then yields

$$
\left|\psi_{k}(M)\right\rangle\left|d_{0}\right\rangle \rightarrow \sum_{i, k_{i}} \alpha_{i k_{i}}^{k}\left|\psi_{k_{i}}\left(M-E_{i}\right)\right\rangle\left|d_{i}\right\rangle
$$

where $\alpha_{i k_{i}}^{k}=\sum_{a, k^{\prime}} c_{i a k^{\prime}}^{k} d_{k_{i}}^{a k^{\prime}}$ and $E_{i}=E_{\mathrm{d}, i} \sqrt{1-2 M l_{\mathrm{P}}^{2} / r_{\mathrm{d}}}$. This represents a microscopic, unitary description of the elementary mining process.

In the description given above, we have separated the detector state from the state of the black hole, but in a treatment fully consistent with the notation in earlier sections, the detector itself must be viewed as excitations over $\left|\psi_{k}(M)\right\rangle$. After the detector response process in eq. (3.34), these excitations can be entangled with Hawking quanta emitted earlier, reflecting the fact that the detector can extract information from the black hole. Since the detector can now be put deep in the zone, in which the Rindler approximation is applicable, this implies that excitations localized within the Rindler wedge corresponding to the region $r>r_{\mathrm{s}}$ are entangled with early Hawking radiation. Does this lead to firewalls as discussed in ref. [6]? The answer is no. The excitations describing the detector are, in the near horizon Rindler approximation, those of modes that are smooth in Minkowski space $(a$ modes in the notation of ref. [6]). Likewise, modes representing negative energy excitations arising from the backreactions are also ones smooth in Minkowski space. Excitations of these modes, of course, do perturb the black hole system, which can indeed be significant if the detector is held very close to the horizon. This effect, however, is caused by physical interactions between the detector and vacuum degrees of freedom, and is confined in the causal future of the interaction event. This is not the firewall phenomenon. 
The semiclassical description of the mining process in eq. (3.36) is obtained by taking maximal mixture for the vacuum indices. Specifically, the semiclassical state before the process starts is given by

$$
\rho(M)=\frac{1}{e^{S_{0}(M)}} \sum_{k=1}^{e^{S_{0}(M)}}\left|\psi_{k}(M)\right\rangle\left|d_{0}\right\rangle\left\langle\psi_{k}(M)\right|\left\langle d_{0}\right| .
$$

The evolution of this state under eq. (3.36) is then

$$
\rho(M) \rightarrow \frac{1}{e^{S_{0}(M)}} \sum_{k=1}^{e^{S_{0}(M)}} \sum_{i, i^{\prime}} \sum_{k_{i}=1}^{e^{S_{0}\left(M-E_{i}\right)}} \sum_{k_{i^{\prime}}^{\prime}=1}^{e^{S_{0}\left(M-E_{i^{\prime}}\right)}} \alpha_{i k_{i}}^{k} \alpha_{i^{\prime} k_{i^{\prime}}^{\prime}}^{k *}\left|\psi_{k_{i}}\left(M-E_{i}\right)\right\rangle\left|d_{i}\right\rangle\left\langle\psi_{k_{i^{\prime}}}\left(M-E_{i^{\prime}}\right)\right|\left\langle d_{i^{\prime}}\right| .
$$

This leads to the density matrix describing the detector state after the process

$$
\rho_{\mathrm{d}}=\sum_{i, i^{\prime}} \gamma_{i i^{\prime}}\left|d_{i}\right\rangle\left\langle d_{i^{\prime}}\right|
$$

where

$$
\gamma_{i i^{\prime}}=\operatorname{Tr}\left[\frac{1}{e^{S_{0}(M)}} \sum_{k=1}^{e^{S_{0}(M)}} \sum_{k_{i}=1}^{e^{S_{0}\left(M-E_{i}\right)}} \sum_{k_{i^{\prime}}^{\prime}=1}^{S_{0}\left(M-E_{i^{\prime}}\right)} \alpha_{i k_{i}}^{k} \alpha_{i^{\prime} k_{i^{\prime}}^{\prime}}^{k *}\left|\psi_{k_{i}}\left(M-E_{i}\right)\right\rangle\left\langle\psi_{k_{i^{\prime}}^{\prime}}\left(M-E_{i^{\prime}}\right)\right]\right] .
$$

Assuming that the microscopic dynamics of the vacuum degrees of freedom are generic, $\gamma_{i i^{\prime}}$ is expected to take the form

$$
\gamma_{i i^{\prime}} \approx \frac{1}{Z} f_{i} e^{-\frac{E_{\mathrm{d}, i}}{T\left(r_{\mathrm{d}}\right)}} \delta_{i i^{\prime}}
$$

where $Z=\sum_{i} f_{i} e^{-E_{\mathrm{d}, i} / T\left(r_{\mathrm{d}}\right)}$, and $f_{i}$ is the detector response function reflecting intrinsic properties of the detector under consideration. This implies that in the semiclassical approximation, the final detector state does not have any information about the original black hole microstate, despite the fact that the fundamental process in eq. (3.36) is, in fact, unitary.

\subsection{The fate of an infalling object}

We now discuss how an object falling into a black hole is described in a distant reference frame. As we have seen, having a well-defined black hole geometry requires a superposition of an enormous number of energy-momentum eigenstates. While the necessary spreads in energy and momentum are small when measured in the asymptotic region, the spreads of local energy and momentum (i.e. those measured by local approximately static observers) are large in the region close to the horizon, because of large gravitational blueshifts. This makes the local temperature $T(r)$ associated with the vacuum degrees of freedom, eq. (3.20), very high near the horizon. We expect that the semiclassical description becomes invalid when this temperature exceeds the string (cutoff) scale, $T(r) \gtrsim 1 / l_{*}$. Namely, semiclassical spacetime exists only in the region

$$
r>r_{\mathrm{s}}=2 M l_{\mathrm{P}}^{2}+O\left(\frac{l_{*}^{2}}{M l_{\mathrm{P}}^{2}}\right)
$$


where $r_{\mathrm{s}}$ is identified as the location of the stretched horizon. The same conclusion can also be obtained by demanding that the gravitational thermal entropy stored in the region where the semiclassical spacetime picture is applicable is a half of the Bekenstein-Hawking entropy, $\mathcal{A} / 8 l_{\mathrm{P}}^{2}$, as discussed in footnote 13 .

Let us consider that an object is dropped from $r=r_{0}$ with vanishing initial velocity, where $r_{0}-2 M l_{\mathrm{P}}^{2} \approx O\left(M l_{\mathrm{P}}^{2}\right)>0$. It then freely falls toward the black hole and hits the stretched horizon at $r=r_{\mathrm{s}}$ in Schwarzschild time of about $4 M l_{\mathrm{P}}^{2} \ln \left(M l_{\mathrm{P}}^{2} / l_{*}\right)$. Before it hits the stretched horizon, the object is described by $a$ and $a_{\text {far }}$, the indices labeling field and string theoretic excitations over the semiclassical background spacetime. After hitting the stretched horizon, the information about the object will move to the index $\bar{a}$, labeling excitations of the stretched horizon. The information about the fallen object will then stay there, at least, for the thermalization (or scrambling) time of the stretched horizon, of order $M l_{\mathrm{P}}^{2} \ln \left(M l_{\mathrm{P}}\right)$. This allows for avoiding the inconsistency of quantum cloning in black hole physics $[13,14]$. Finally, the information in $\bar{a}$ will further move to $k$, which can (later) be extracted by an observer in the asymptotic region via the Hawking emission or mining process, as described in the previous two subsections.

We note that the statement that an object is in the semiclassical regime (i.e. represented by indices $a$ and $a_{\text {far }}$ ) does not necessarily mean that it is well described by semiclassical field theory. Specifically, it is possible that stringy effects become important before the object hits the stretched horizon. As an example, consider dropping an elementary particle of mass $m\left(\ll 1 / l_{*}\right)$ from $r=r_{0}$ with zero initial velocity. (Here, by elementary we mean that there is no composite structure at lengthscale larger than $l_{*}$.) The local energy and local radial momentum of the object will then vary, as it falls, as:

$$
E_{\mathrm{loc}}=m \sqrt{\frac{1-\frac{2 M l_{\mathrm{P}}^{2}}{r_{0}}}{1-\frac{2 M l_{\mathrm{P}}^{2}}{r}}}, \quad \quad p_{\mathrm{loc}}=-m \sqrt{\frac{\frac{2 M l_{\mathrm{P}}^{2}}{r}-\frac{2 M l_{\mathrm{P}}^{2}}{r_{0}}}{1-\frac{2 M l_{\mathrm{P}}^{2}}{r}}} .
$$

The values of $E_{\text {loc }} \approx-p_{\text {loc }}$ get larger as $r$ gets smaller, and for $m \gg 1 / M l_{\mathrm{P}}^{2}$ (which we assume here) become of order $1 / l_{*}$ before the object hits the stretched horizon, i.e. at

$$
r-2 M l_{\mathrm{P}}^{2} \simeq 2 M l_{\mathrm{P}}^{2}\left(m l_{*}\right)^{2}\left(1-\frac{2 M l_{\mathrm{P}}^{2}}{r_{0}}\right) .
$$

The Schwarzschild time it takes for the object to reach this point is only about $-4 M l_{\mathrm{P}}^{2} \ln \left(m l_{*}\right)$, much smaller than the time needed to reach the stretched horizon, $4 M l_{\mathrm{P}}^{2} \ln \left(M l_{\mathrm{P}}^{2} / l_{*}\right)$. After the object reaches this point, i.e. when $E_{\mathrm{loc}} \approx-p_{\text {loc }} \gtrsim 1 / l_{*}$, stringy effects might become important; specifically, its Lorentz contraction saturates and transverse size grows with $E_{\text {loc }}[38]$. Note that this dependence of the description on the boost of a particle does not necessarily mean violation of Lorentz invariance - physics can still be fully Lorentz invariant. ${ }^{18}$

\footnotetext{
${ }^{18}$ It is illuminating to consider how these stringy effects appear in a two-particle scattering process in Minkowski space. For $\sqrt{s} \lesssim 1 / l_{*}$, where $s$ is the Mandelstam variable, there is a reference frame in which energies/momenta of both particles are smaller than $1 / l_{*}$, guaranteeing that these effects are not important in the process. For $\sqrt{s}>1 / l_{*}$, on the other hand, at least one particle has an energy/momentum larger than $1 / l_{*}$ in any reference frame, suggesting that stringy effects become important in scattering with such high $\sqrt{s}$.
} 


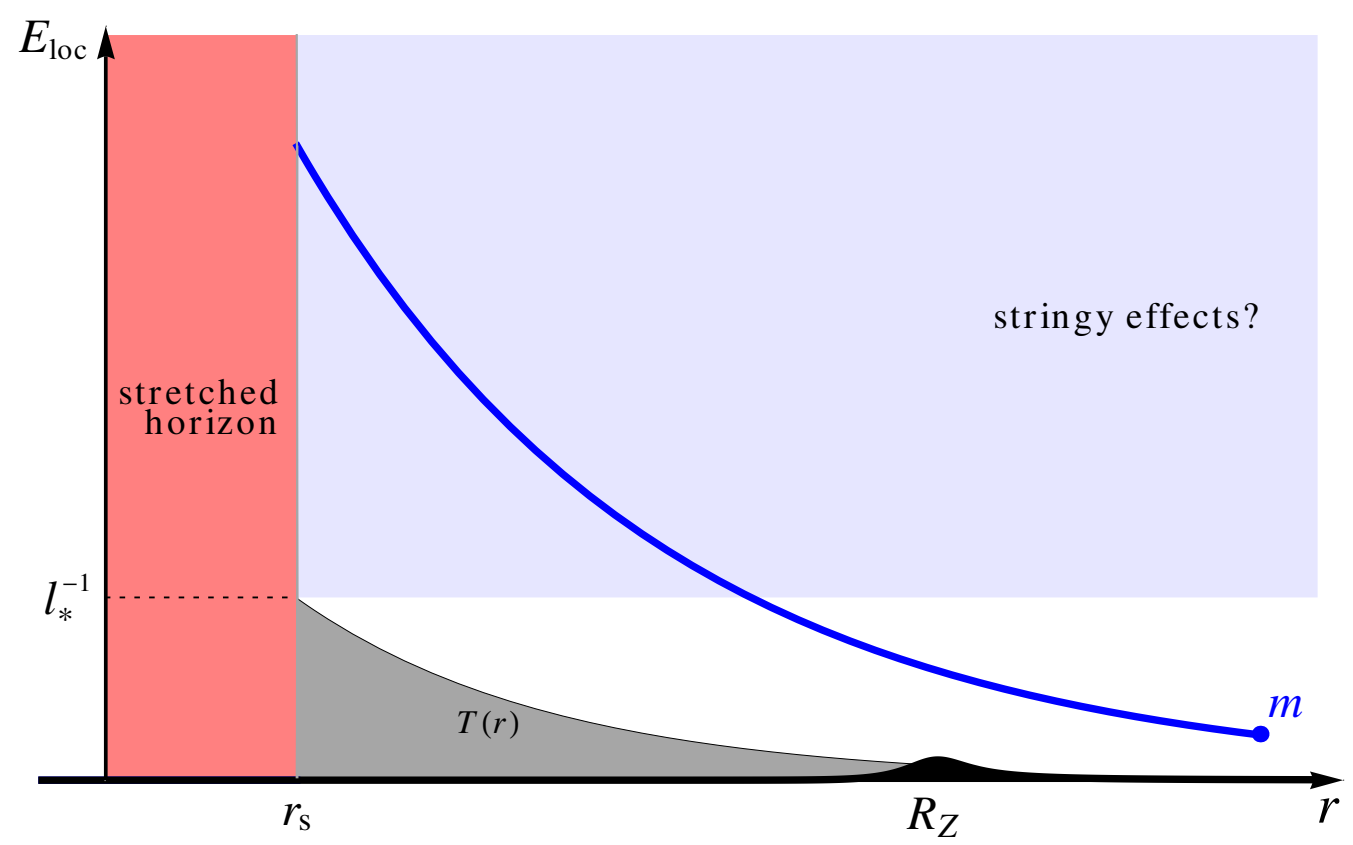

Figure 3. A schematic depiction of the fate of an elementary particle of mass $m\left(1 / M l_{\mathrm{P}}^{2} \ll m \ll\right.$ $\left.1 / l_{*}\right)$ dropped into a black hole, viewed in a distant reference frame. As the particle falls, its local energy blueshifts and exceeds the string/cutoff scale $1 / l_{*}$ before it hits the stretched horizon. After this point, stringy effects could become important, although the semiclassical description of the object may still be applicable. The object hits the stretched horizon at a Schwarzschild time of about $4 M l_{\mathrm{P}}^{2} \ln \left(M l_{\mathrm{P}}^{2} / l_{*}\right)$ after the drop. After this time, the semiclassical description of the object is no longer applicable, and the information about the object will be encoded in the index $\bar{a}$, representing excitations of the stretched horizon. (This information will further move to the vacuum index $k$ later, so that it can be extracted by an observer in the asymptotic region via the Hawking emission or mining process.)

A schematic picture for the fate of an infalling object described above is given in figure 3. In a distant reference frame, the semiclassical description of the object is applicable only until it hits the stretched horizon, after which it is represented as excitations of the stretched horizon. On the other hand, according to general relativity (or the equivalence principle), the falling object does not experience anything other than smooth empty spacetime when it crosses the horizon, except for effects associated with curvature, which are very small for a black hole of mass $M \gg 1 / l_{\mathrm{P}}$. If this picture is correct, then we expect there is a way to reorganize the dynamics of the stretched horizon such that the general relativistic smooth interior of the black hole becomes manifest. In the complementarity picture, this is achieved by performing an appropriate reference frame change. We now move on to discuss this issue.

\section{Black hole - An infalling description}

In order to describe the fate of an infalling object using low energy language after it crosses the Schwarzschild horizon, we need to perform a change of the reference frame from 
a distant one, which we have been considering so far, to an infalling one which falls into the black hole with the object. In general, studying this issue is complicated by the fact that the general and precise formulation of complementarity is not yet known, but we may still explore the expected physical picture based on some general considerations.

The aim of this section is to argue that the existence of interior spacetime, as suggested by general relativity, does not contradict the unitarity of the Hawking emission and black hole mining processes, as described in the previous section in a distant reference frame. We do this by first arguing that there exists a reference frame - an infalling reference frame - in which the spacetime around a point on the Schwarzschild horizon appears as a large nearly flat region, with the curvature lengthscale of order $M l_{\mathrm{P}}^{2}$. This is a reference frame whose origin falls freely from rest from a point sufficiently far from the black hole. We discuss how the description based on this reference frame is consistent with that in the distant reference frame, despite the fact that they apparently look very different, for example in spacetime locations of the vacuum degrees of freedom.

We then discuss how the system is described in more general reference frames, in particular a reference frame whose origin falls from rest from a point close to the Schwarzschild horizon. We will also discuss (non-)relations of black hole mining by a near-horizon static detector and the - seemingly similar — Unruh effect in Minkowski space. The discussion in this section illuminates how general coordinate transformations may work at the level of full quantum gravity, beyond the approximation of quantum field theory in curved spacetime.

\subsection{Emergence of interior spacetime - Free fall from a distance}

What does a reference frame really mean? According to the general complementarity picture described in section 2 , it corresponds to a foliation of a portion of spacetime which a single (hypothetical) observer can access. As discussed there, the procedure to erect such a reference frame should not depend on the background geometry in order for the framework to be applicable generally, and there is currently no precise, established formulation to do that (although there are some partially successful attempts; see, e.g., ref. [20]). Here we focus only on classes of reference frames describing the same system with a fixed black hole background. This limitation allows us to bypass many of the issues arising when we consider the most general application of the complementarity picture.

In this subsection, we consider a class of reference frames which we call infalling reference frames. We argue that a reference frame in this class makes it manifest that the spacetime near the origin of the reference frame appears as a large approximately flat region when it crosses the Schwarzschild horizon, up to corrections from curvature of lengthscale $M l_{\mathrm{P}}^{2}$. We discuss how the interior spacetime of the black hole can emerge through the complementarity transformation representing a change of reference frame from the distant to infalling ones. Consistency of the infalling picture described here with the distant frame description in section 3 will be discussed in more detail in the next subsection.

We consider a reference frame associated with a freely falling (local Lorentz) frame, with its spatial origin $p_{0}$ following the worldline representing a hypothetical observer $[15$, 20]. In particular, we let the origin of the reference frame, $p_{0}$, follow the trajectory of a timelike geodesic, representing the observer who is released from rest at $r=r_{0}$, with 
$r_{0}$ sufficiently far from the Schwarzschild horizon, $r_{0}-2 M l_{\mathrm{P}}^{2} \gtrsim M l_{\mathrm{P}}^{2}$. According to the complementarity hypothesis, the system described in this reference frame does not have a (hot) stretched horizon at the location of the Schwarzschild horizon when $p_{0}$ crosses it. (The stretched horizon must have existed around the Schwarzschild horizon when $p_{0}$ was far away, $r_{p_{0}}-2 M l_{\mathrm{P}}^{2} \gtrsim O\left(M l_{\mathrm{P}}^{2}\right)$, because the description in those earlier times must be approximately that of a distant reference frame, i.e. that discussed in the previous section.) In particular, the region around $p_{0}$ must appear approximately flat, i.e. up to small effects from curvature of order $1 / M^{2} l_{\mathrm{P}}^{4}$, until $p_{0}$ approaches the singularity.

In this infalling description, we expect that a "horizon" signaling the breakdown of the semiclassical description lies in the directions associated with "past-directed and inward" light rays (the directions with increasing $r$ and decreasing $t$ after $p_{0}$ crosses $r=2 M l_{\mathrm{P}}^{2}$ ) as viewed from $p_{0}$; see figure $4 .{ }^{19}$ As in the stretched horizon in a distant reference frame, this "horizon" emerges because of the "squeezing" of equal-time hypersurfaces; in particular, an observer following the trajectory of $p_{0}$ may probe only a tiny region near the Schwarzschild horizon for signals arising from this surface. (Note that $-r$ plays a role of time inside the Schwarzschild horizon.) Considering angular directions, this "horizon" has an area of order $M^{2} l_{\mathrm{P}}^{4}$, and can be regarded as being located at distances of order $M l_{\mathrm{P}}^{2}$ away from $p_{0}$ (with an appropriately defined distance measure on generic equal-time hypersurfaces in the infalling reference frame; see section 4.2).

In analogy with the case of a distant frame description, we denote basis states for the general microstates in an infalling reference frame (before $p_{0}$ reaches the singularity) as

$$
\left|\Psi_{\bar{\alpha} \alpha \alpha_{\mathrm{far} ; \kappa}}(M)\right\rangle
$$

where $\bar{\alpha}$ labels the excitations of the "horizon," and $\alpha$, and $\alpha_{\mathrm{far}}$ are the indices labeling the semiclassical excitations near and far from the black hole, conveniently defined; $\kappa$ is the vacuum index in an infalling reference frame, representing degrees of freedom that cannot be resolved by semiclassical operators. ${ }^{20}$ The complementarity transformation provides a map from the basis states in a distant description, eq. (3.4), to those in an infalling description, eq. (4.1), and vice versa. The general form of this transformation can be quite complicated, depending, e.g., on equal-time hypersurfaces taken in the two descriptions (which are in turn related with the general procedure of erecting reference frames by standard coordinate transformations within each causal patch). Here we consider how various indices are related under the transformation, focusing on the near black hole region.

Imagine that equal-time hypersurfaces in the two - distant and infalling - reference frames agree at some time $t=t_{0}$ in the spacetime region near but outside the surface where the stretched horizon exists if viewed from the distant reference frame. (Note that the stretched horizon has physical substance only in a distant reference frame.) We are interested in how basis states in the two descriptions transform between each other in

\footnotetext{
${ }^{19}$ This "horizon," as viewed from an infalling reference frame, should not be confused with the stretched, or Schwarzschild, horizon as viewed from a distant reference frame.

${ }^{20}$ After $p_{0}$ hits the singularity, the system as viewed from the infalling reference frame can only be represented by "singularity states": intrinsically quantum gravitational states that do not allow for a spacetime interpretation [15].
} 


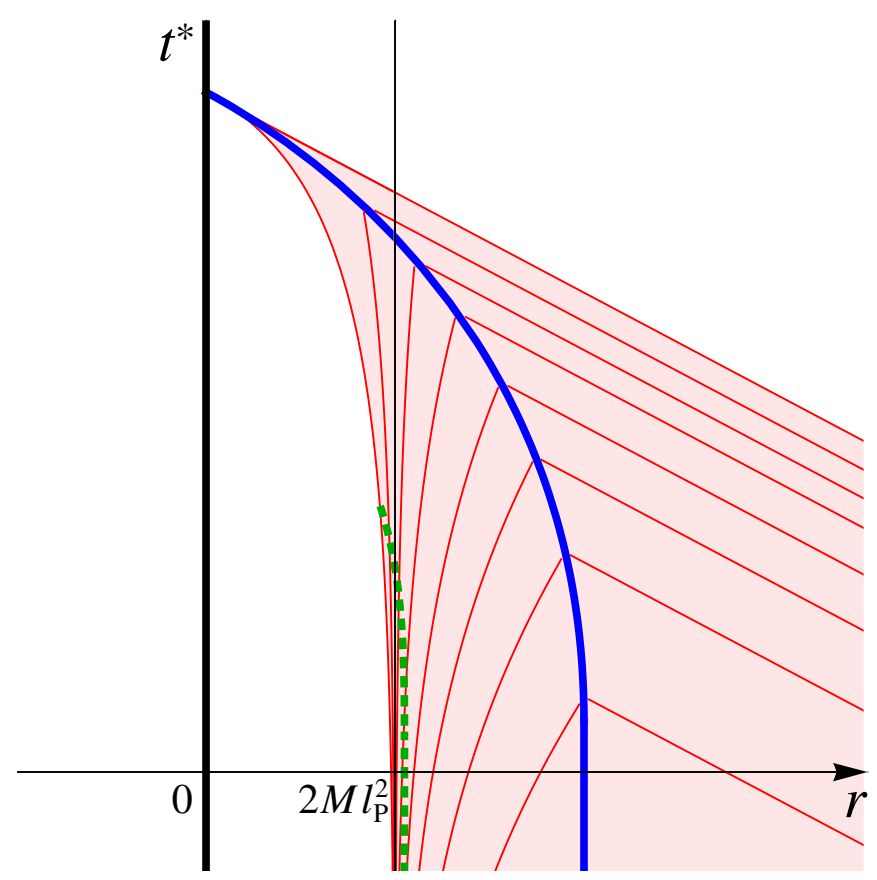

Figure 4. A sketch of an infalling reference frame in an Eddington-Finkelstein diagram: the horizontal and vertical axes are $r$ and $t^{*}=t+r^{*}-r$, respectively, where $r^{*}$ is the tortoise coordinate. The thick (blue) line denotes the spacetime trajectory of the origin, $p_{0}$, of the reference frame, while the thin (red) lines represent past-directed light rays emitted from $p_{0}$. The shaded area is the causal patch associated with the reference frame, and the dotted (green) line represents the stretched "horizon" as viewed from this reference frame.

the timescale of the fall of the infalling reference frame. The time here can be taken as the proper time at $p_{0}$ in each reference frame $[15,20]$, which is approximately the Schwarzschild time for the distant reference frame. In this case, the relevant timescale is $t-t_{0} \lesssim O\left(M l_{\mathrm{P}}^{2} \ln \left(M l_{\mathrm{P}}\right)\right)$ in the distant reference frame, while $t-t_{0} \lesssim O\left(M l_{\mathrm{P}}^{2}\right)$ in the infalling reference frame.

As discussed in section 3.6, in the distant reference frame, an object dropped from some $r_{0}$ with $r_{0}-2 M l_{\mathrm{P}}^{2} \approx O\left(M l_{\mathrm{P}}^{2}\right)$ is first represented by $a$ and then by $\bar{a}$ after it hits the stretched horizon. On the other hand, in the infalling frame, the object is represented by the index $\alpha$ throughout, first as a semiclassical excitation outside the Schwarzschild horizon and then as a semiclassical excitation inside the Schwarzschild horizon, implying that the object does not find anything special at the horizon. Here, we have assumed that $p_{0}$ follows (approximately) the trajectory of the falling object. This suggests that a portion of the $\alpha$ index representing excitations in the interior of the black hole is transformed into the $\bar{a}$ index in the distant description (and vice versa) under the complementarity transformation; i.e., the interior of the black hole accessible from the infalling reference frame is encoded in the excitations of the stretched horizon in the distant reference frame. Note that the amount of information needed to reconstruct the interior (in the semiclassical sense) is much smaller than the Bekenstein-Hawking entropy $[23,24]$ — the logarithm of the dimension of the relevant Hilbert space is of order $\left(\mathcal{A} / l_{\mathrm{P}}^{2}\right)^{q}$ with $q<1$. 
In the exterior spacetime region, the portion of the $\alpha$ index representing excitations there, as well as the $\alpha_{\text {far }}$ index, are mapped to the corresponding $a$ and $a_{\text {far }}$ indices, and vice versa (after matching the equal-time hypersurface in the two descriptions through appropriate time evolutions). Because equal-time hypersurfaces foliate the causal patch, excitations in the far exterior region naturally have trans-Planckian energies in the infalling description. However, as discussed in section 3.6, this does not mean that the semiclassical description is invalid - objects may still be described as excitations in the semiclassical spacetime, although stringy effects may become important. Indeed, we expect that the semiclassical description is applicable in the far exterior region even in the infalling reference frame, because of the absence of the "squeezing" effect described above which leads to the breakdown of the semiclassical picture.

We emphasize that the construction of the interior spacetime described here does not suffer from the paradoxes discussed in refs. [6-8]. By labeling states in terms of excitations, we are in a sense representing the interior spacetime already in the distant description. (The interpretation, however, is different. In the distant description, the relevant excitations must be regarded as those of the stretched horizon.) In fact, we do not find any inconsistency in postulating that the dynamics of an infalling object is described by the corresponding Hamiltonian in the semiclassical theory in a sufficiently small region around $p_{0}$, to the extent that microscopic details of interactions with $\kappa$ degrees of freedom are neglected. Namely, we do not find any inconsistency in postulating that physics at the classical level is well described by general relativity.

Finally, we discuss where the fine-grained vacuum degrees of freedom represented by $\kappa$ must be viewed as being located in the infalling description. Because of the lack of an obvious static limit, it is not straightforward to answer to this question. Nevertheless, it seems natural to expect, in analogy with the case of a distant description, that most of the degrees of freedom are located close to the "horizon" (in terms of a natural distance measure in which the distance between the "horizon" and $p_{0}$ is of order $M l_{\mathrm{P}}^{2}$ ). In fact, we expect that the number of $\kappa$ degrees of freedom existing around $p_{0}$ within a distance scale sufficiently smaller than $M l_{\mathrm{P}}^{2}$ is of $O(1)$ or smaller, since the time and length scales of the system characterizing local deviations from Minkowski space (as viewed from the infalling reference frame) are both of order $M l_{\mathrm{P}}^{2}$. As in the case of the distant description, we expect that the $\kappa$ degrees of freedom do not extend significantly to the far exterior region, since the existence of the black hole does not affect the spacetime there much. ${ }^{21}$

\subsection{Consistency between the distant and infalling descriptions}

In analyzing a black hole system in a distant reference frame, we argued that the microscopic information about the black hole, represented by the $k$ index, is distributed according

\footnotetext{
${ }^{21}$ Note that the descriptions in the two reference frames are already different at the semiclassical level. For example, the backreaction of a detector click in a distant reference frame is described as an absorption of a particle in the thermal bath, while in an infalling reference frame it is described as an emission of a particle, with the difference arising from different definitions of energy in the two reference frames [39]. The reference frame dependence discussed here is much more drastic, however - the spacetime locations of physical degrees of freedom are different in the two reference frames.
} 
to the gravitational thermal entropy calculated using semiclassical field theory. In particular, on the Schwarzschild (or stretched) horizon, this information has a Planckian density: one qubit per area of order $l_{\mathrm{P}}^{2}$ on the horizon (or per volume of order $l_{\mathrm{P}}^{3}$ if we take into account the "thickness" of the stretched horizon, $\left.\sim l_{\mathrm{P}}\right)$. On the other hand, we have just argued that in an infalling reference frame, the spacetime distribution of the microscopic information (now represented by the $\kappa$ index) is different. In particular, the spatial density of the information around the Schwarzschild horizon, when the origin of the reference frame passes through it, is very small: one qubit per volume of order $\left(M l_{\mathrm{P}}^{2}\right)^{3}$. How can we reconcile these two seemingly very different perspectives?

In this subsection, we consider this problem and argue that despite the fact that the spacetime distribution of the microscopic information depends on the reference frame one chooses to describe the system, the answers to any operationally well-defined question one obtains in different reference frames are consistent with each other. As an example most relevant to our discussion, we consider a physical detector hovering at a constant Schwarzschild radius $r=r_{\mathrm{d}}\left(>2 M l_{\mathrm{P}}^{2}\right)$. In a distant description, the spatial density of the microscopic information, represented by $k$, is large at the location of the detector when $r_{\mathrm{d}}-2 M l_{\mathrm{P}}^{2} \ll M l_{\mathrm{P}}^{2}$. Such a detector (or a system of detectors) can thus be used for black hole mining: accelerated extraction of energy and information from the black hole. In an infalling reference frame, however, the density of the microscopic information, represented by $\kappa$, is very small at the detector location, at least when the origin of the reference frame, $p_{0}$, passes nearby. This implies that the rate of extracting information from spacetime cannot be much faster than $1 / M l_{\mathrm{P}}^{2}$ around $p_{0}$ in the infalling description, reflecting the fact that the spacetime appears approximately flat there. How are these two descriptions consistent?

In the distant description, the rate of extracting microscopic information about the black hole is at most of order one qubit per Schwarzschild time $1 / T_{\mathrm{H}}=8 \pi M l_{\mathrm{P}}^{2}$ per channel, regardless of the location of the detector [33] — the acceleration of information extraction occurs not because of a higher speed of information extraction in each channel but because of an increased number of channels available by immersing the detector deep into the zone. This implies that each single detector, which we define to act on a single channel, "clicks" once (i.e. extracts of $O(1)$ qubits) per a Schwarzschild time of order $8 \pi M l_{\mathrm{P}}^{2}$.

Now, consider describing such a detector in an infalling reference frame whose origin $p_{0}$ is released at $r=2 M l_{\mathrm{P}}^{2}+O\left(M l_{\mathrm{P}}^{2}\right)$ from rest, at an angular location close to the detector. To understand the relevant kinematics, we adopt the near-horizon Rindler approximation: for $r>2 M l_{\mathrm{P}}^{2}$

$$
\rho \approx 2 \sqrt{2 M l_{\mathrm{P}}^{2}\left(r-2 M l_{\mathrm{P}}^{2}\right)}, \quad \omega \approx \frac{t}{4 M l_{\mathrm{P}}^{2}},
$$

in terms of which the metric is given by

$$
d s^{2} \approx-\rho^{2} d \omega^{2}+d \rho^{2}+r(\rho)^{2} d \Omega .
$$

As is well-known, this metric can be written in the Minkowski form

$$
d s^{2} \approx-d T^{2}+d Z^{2}+r(T, Z)^{2} d \Omega,
$$


by introducing the coordinates

$$
T=\rho \sinh \omega, \quad Z=\rho \cosh \omega,
$$

which can be extended into the $r<2 M l_{\mathrm{P}}^{2}$ region. Our setup corresponds to the situation in which the detector follows a trajectory of a constant $\rho$ :

$$
\rho=\rho_{\mathrm{d}} \ll M l_{\mathrm{P}}^{2},
$$

while the origin of the reference frame $p_{0}$ — or the (fictitious) observer — is at a constant $Z$ :

$$
Z=Z_{\mathrm{o}} \approx O\left(M l_{\mathrm{P}}^{2}\right)
$$

Note that while we approximate the geometry by flat space, given by eq. (4.3) or (4.4), the actual system has small nonzero curvature with lengthscale of order $M l_{\mathrm{P}}^{2}$.

As discussed above, the detector extracts an $O(1)$ amount of information in each time interval of

$$
\Delta \omega \approx O\left(\frac{1}{4 M l_{\mathrm{P}}^{2} T_{H}}\right) \approx O(1)
$$

while the "observer," $p_{0}$, and the detector meet (or pass by each other) at

$$
\left(\begin{array}{c}
\omega \\
\rho
\end{array}\right)=\left(\begin{array}{c}
\operatorname{arccosh} \frac{Z_{\mathrm{o}}}{\rho_{\mathrm{d}}} \\
\rho_{\mathrm{d}}
\end{array}\right) \equiv\left(\begin{array}{c}
\omega_{*} \\
\rho_{*}
\end{array}\right) .
$$

This implies that in the Minkowski coordinates - i.e. as viewed from the infalling observer $p_{0}$ — the detector clicks only once in each time/space interval of

$$
\begin{aligned}
& \left.\Delta T \approx \Delta \omega \frac{\partial T}{\partial \omega}\right|_{\omega=\omega_{*}, \rho=\rho_{*}} \approx Z_{\mathrm{o}} \approx O\left(M l_{\mathrm{P}}^{2}\right), \\
& \left.\Delta Z \approx \Delta \omega \frac{\partial Z}{\partial \omega}\right|_{\omega=\omega_{*}, \rho=\rho_{*}} \approx Z_{\mathrm{o}} \approx O\left(M l_{\mathrm{P}}^{2}\right),
\end{aligned}
$$

around $p_{0}$. This is precisely what we expect from the equivalence principle: the spacetime appears approximately flat when viewed from an infalling observer, up to curvature effects with lengthscale of $M l_{\mathrm{P}}^{2}$. While the detector clicks of order $\ln \left(M l_{\mathrm{P}}\right)$ times within the causal patch of the infalling reference frame, all these clicks occur at distances of order $M l_{\mathrm{P}}^{2}$ away from $p_{0}$, where we expect a higher density of $\kappa$ degrees of freedom. The two descriptions - distant and infalling - are therefore consistent, despite the fact that the spacetime distributions of the microscopic information about the black hole - represented by $k$ and $\kappa$, respectively - are different in the two reference frames.

While we have so far discussed the case in which a physical detector is located close to the Schwarzschild horizon, the conclusion is the same in the case of spontaneous Hawking emission. In this case, since Hawking particles appear as semiclassical excitations only at $r-2 M l_{\mathrm{P}}^{2} \gtrsim M l_{\mathrm{P}}^{2}$ with local energies of order $1 / M l_{\mathrm{P}}^{2}$, the consistency of the two descriptions is in a sense obvious. Alternatively, one can regard this case as the $\rho_{\mathrm{d}} \approx M l_{\mathrm{P}}^{2}$ limit of the previous analysis. While the Rindler approximation is strictly valid only for $\rho$ sufficiently smaller than $M l_{\mathrm{P}}^{2}$, qualitative results are still valid for $\rho_{\mathrm{d}} \approx M l_{\mathrm{P}}^{2}$; in particular, the estimates in eqs. (4.10), (4.11) are valid at an order of magnitude level. 


\subsection{Other reference frames - Free fall from a nearby point}

In this subsection, we consider how the black hole is described in a class of reference frames whose origin follows a timelike geodesic released from rest at $r=r_{0}$, where $r_{0}$ is close to the Schwarzschild horizon, $r_{0}-2 M l_{\mathrm{P}}^{2} \ll M l_{\mathrm{P}}^{2}{ }^{22}$ We argue that the description in these reference frames does not look similar to either the distant or infalling description discussed before, and yet it is consistent with both of them. ${ }^{23}$

To understand how the black hole appears in such a reference frame, let us consider a setup similar to that in section 4.2 - a physical detector hovering at a constant Schwarzschild radius $r=r_{\mathrm{d}}$ - and see how this system is described in the reference frame. As in section 4.2, we may adopt the Rindler approximation, in which eq. (4.7) is now replaced by

$$
Z=Z_{\mathrm{o}} \ll M l_{\mathrm{P}}^{2}
$$

This implies that as viewed from this reference frame, the detector clicks once in each time/space interval of

$$
\Delta T \approx \Delta Z \approx Z_{\mathrm{o}} \ll M l_{\mathrm{P}}^{2}
$$

Here, we have assumed that $\rho_{\mathrm{d}}<Z_{\mathrm{o}}$. Since each detector click extracts an $O(1)$ amount of information from spacetime, which we expect not to occur in Minkowski space, this implies that the spacetime cannot be viewed as approximately Minkowski space over a region beyond lengthscale $Z_{\mathrm{o}}$. In particular, in contrast with the case in an infalling reference frame (with $Z_{\mathrm{o}} \gtrsim O\left(M l_{\mathrm{P}}^{2}\right)$ ), the spacetime region around $p_{0}$ in this reference frame does not appear nearly flat over lengthscale of $M l_{\mathrm{P}}^{2}$ when $p_{0}$ crosses the Schwarzschild horizon.

At a technical level, this difference arises from the fact that the relative boost of $p_{0}$ with respect to the distant reference frame when $p_{0}$ approaches the detector

$$
\gamma=\frac{1}{\sqrt{1-v_{\mathrm{rel}}^{2}}}=\sqrt{\frac{1-\frac{2 M l_{\mathrm{P}}^{2}}{r_{0}}}{1-\frac{2 M l_{\mathrm{P}}^{2}}{r_{d}}}},
$$

is very different in the two reference frames. In an infalling reference frame $\gamma$ is huge, $\approx O\left(M l_{\mathrm{P}}^{2} / \rho_{\mathrm{d}}\right)$, while in the reference frame considered here $\gamma \approx O\left(Z_{\mathrm{o}} / \rho_{\mathrm{d}}\right)$, which is not as large as that in the infalling case. In the infalling reference frame of sections 4.1 and 4.2, the huge boost of $\gamma \approx O\left(M l_{\mathrm{P}}^{2} / \rho_{\mathrm{d}}\right)$ "stretched" the interval between detector clicks to time/length scales of order $M l_{\mathrm{P}}^{2}$. Here, this "stretching" makes only a small region around $p_{0}$, with lengthscale of order $Z_{\mathrm{o}}\left(\ll M l_{\mathrm{P}}^{2}\right)$, look nearly flat at any given time.

We may interpret this result to mean that in the reference frame under consideration, the "horizon" (as viewed from this reference frame) is located at a distance of order $Z_{\mathrm{o}}$ away from $p_{0}$, so that detector clicks occur near or "on" this surface. (In the latter

\footnotetext{
${ }^{22}$ In a full geometry in which the black hole is formed by collapsing matter, the trajectory of the origin, $p_{0}$, of such a reference frame corresponds to a fine-tuned one in which $p_{0}$ stays near outside of the Schwarzschild horizon for long time due to large outward velocities at early times. (Here, we have focused only on the relevant branch in the full quantum state; see, e.g., footnote 4.)

${ }^{23}$ Note that we use the term "infalling reference frame" exclusively for reference frames discussed in sections 4.1 and 4.2 , i.e. the ones in which $p_{0}$ starts from rest at $r_{0}$ with $r_{0}-2 M l_{\mathrm{P}}^{2} \gtrsim O\left(M l_{\mathrm{P}}^{2}\right)$.
} 
case, the detector click events must be viewed as occurring in the regime outside the applicability of the semiclassical description; in particular, they can only be described as complicated quantum gravitational processes occurring on the "horizon.") Since we expect that microscopic information about the black hole (analogous to $k$ and $\kappa$ in the distant and infalling reference frames, respectively) is located near and on the "horizon," there is no inconsistency that detector clicks extract microscopic information from the black hole.

One might be bothered by the fact that in this reference frame spacetime near the Schwarzschild horizon does not appear large, $\approx O\left(M l_{\mathrm{P}}^{2}\right)$, nearly flat space, and consider that this implies the non-existence of a large black hole interior as suggested by general relativity. This is, however, not correct. The existence of $a$ reference frame in which spacetime around the Schwarzschild horizon appears as a large nearly flat region - in particular, the existence of an infalling reference frame discussed in sections 4.1 and 4.2 - already ensures that an infalling physical object/observer does not experience anything special, e.g. firewalls, when it/he/she crosses the Schwarzschild horizon. The analysis given here simply says that the spacetime around the Schwarzschild horizon does not always appear as a large nearly flat region, even in a reference frame whose origin falls freely into the black hole. This extreme relativeness of descriptions is what we expect from complementarity.

\section{4 (Non-)relations with the Unruh effect in Minkowski space}

It is often thought that the system described above is similar to an accelerating detector existing in Minkowski space, based on a similarity of geometries between the two setups. If this were true at the full quantum level, it would mean that the description in an inertial reference frame in Minkowski space must possess a "horizon," at which the semiclassical description of the system breaks down. Does this make sense?

Here we argue that physics of a detector held near the Schwarzschild horizon, given above in section 4.3, is, in fact, different from that of an accelerating detector in Minkowski space. The intuition that the two must be similar comes from the (wrong) perception that the detector located near the Schwarzschild horizon feels a high blueshifted Hawking temperature, $\approx 1 / \rho_{\mathrm{d}} \gg 1 / M l_{\mathrm{P}}^{2}$, which makes the detector click at a high rate, while the spacetime curvature there is very small, with lengthscale $\approx M l_{\mathrm{P}}^{2}$, so that such a tiny curvature must not affect the system. This intuition, however, is flawed by mixing up two different pictures - the system as viewed at the location of the detector and as viewed in the asymptotic region.

Suppose we represent all quantities as defined in the asymptotic region. The temperature a detector feels is then of order $1 / M l_{\mathrm{P}}^{2}$ and the timescale for detector clicks is $T \approx O\left(M l_{\mathrm{P}}^{2}\right)$ for any $r_{\mathrm{d}}>2 M l_{\mathrm{P}}^{2}$. On the other hand, the energy density of the black hole region is of order $M /\left(M l_{\mathrm{P}}^{2}\right)^{3}$, so that the curvature lengthscale $L$ is estimated as

$$
\frac{1}{L^{2}} \sim G_{\mathrm{N}} \frac{M}{\left(M l_{\mathrm{P}}^{2}\right)^{3}} \sim \frac{1}{\left(M l_{\mathrm{P}}^{2}\right)^{2}} .
$$

This implies that

$$
T \sim L \sim O\left(M l_{\mathrm{P}}^{2}\right)
$$


namely, curvature is expected to give an $O(1)$ effect on the dynamics of the detector response.

The same conclusion can also be reached when we represent all the quantities in the static frame at the detector location. In this case, the temperature the detector feels is of order $1 / M l_{\mathrm{P}}^{2} \chi$, where $\chi=\sqrt{1-2 M l_{\mathrm{P}}^{2} / r_{\mathrm{d}}}$ is the redshift factor, so that $T \approx O\left(M l_{\mathrm{P}}^{2} \chi\right)$. On the other hand, the energy density of the black hole region is given by $\sim(M / \chi) /\left(M l_{\mathrm{P}}^{2}\right)^{3} \chi$, so that the "blueshifted curvature length" $L$ is given by

$$
\frac{1}{L^{2}} \sim G_{\mathrm{N}} \frac{M / \chi}{\left(M l_{\mathrm{P}}^{2}\right)^{3} \chi} \sim \frac{1}{\left(M l_{\mathrm{P}}^{2} \chi\right)^{2}}
$$

This yields

$$
T \sim L \sim O\left(M l_{\mathrm{P}}^{2} \chi\right)
$$

again implying that curvature provides an $O(1)$ effect on the dynamics.

It is, therefore, no surprise that the physics of a near-horizon detector in section 4.3 differs significantly from that of an accelerating detector in Minkowski space experiencing the Unruh effect [28]. In fact, we consider, as we naturally expect, that an inertial frame description in Minkowski space does not have a horizon, implying that no information about spacetime is extracted by an accelerating detector, despite the fact that it clicks at a rate controlled by the acceleration $a, T \approx O(1 / a)$, in the detector's own frame. This is indeed consistent with the idea that any information must be accompanied by energy. In the black hole case, the detector mines the black hole, i.e. its click extracts energy from the black hole spacetime, while in the Minkowski case the energy needed to excite the detector comes entirely from the force responsible for the acceleration of the detector the detector does not mine energy from Minkowski space. We conclude that blueshifted Hawking radiation and Unruh radiation in Minkowski space are very different as far as the information flow is concerned.

Does this imply a violation of the equivalence principle? The equivalence principle states that gravity is the same as acceleration, and the above statement might seem to contradict this principle. This is, however, not true. The principle demands the equivalence of the two only at a point in space in a given coordinate system, and the descriptions of the two systems discussed above - a black hole and Minkowski space - are indeed the same in an infinitesimally small (or lengthscale of order $l_{*}$ ) neighborhood of $p_{0}$. The principle does not require that the descriptions must be similar in regions away from $p_{0}$, and indeed they are very different: there is a "horizon" at a distance of order $Z_{\mathrm{o}}$ from $p_{0}$ in the black hole case while there is no such thing in the Minkowski case. And it is precisely in these regions that the detector clicks to extract (or non-extract) information from the black hole (Minkowski) spacetime. In quantum mechanics, a system is specified by a quantum state which generally encodes global information on the equal-time hypersurface. It is, therefore, natural that the equivalence principle, which makes a statement only about a point, does not enforce the equivalence between physics of blueshifted Hawking radiation and of the Unruh effect in Minkowski space at the fully quantum level. 


\subsection{Complementarity: general covariance in quantum gravity}

We have argued that unitary information transfer described in section 3, associated with Hawking emission and black hole mining, is consistent with the existence of the interior spacetime suggested by general relativity. We can summarize important lessons we have learned about quantum gravity through this study in the following three points:

- In a fixed reference frame, the microscopic information about spacetime, in this case about a black hole, may be viewed as being associated with specific spacetime locations. In particular, for a (quasi-)static description of a system, these degrees of freedom are distributed according to the gravitational thermal entropy calculated using semiclassical field theory. The distribution of these degrees of freedom - which we may call "constituents of spacetime" - controls how they can interact with the degrees of freedom in semiclassical theory, e.g. matter and radiation in semiclassical field theory.

- The spacetime distribution of the microscopic information, however, changes if we adopt a different reference frame to describe the system. In this sense, the "constituents of spacetime" are not anchored to spacetime; they are associated with specific spacetime locations only after the reference frame is fixed. In particular, no reference frame independent statement can be made about where these degrees of freedom are located in spacetime. We may view this as a manifestation of the holographic principle $[23,40,41]$ - gauge invariant degrees of freedom in a quantum theory of gravity live in some "holographic space."

- Despite the strong reference frame dependence of the location of the microscopic degrees of freedom, the answers to any physical question are consistent with each other when asked in different reference frames. In particular, when we change the reference frame, the distribution of the microscopic degrees of freedom (as well as some of the semiclassical degrees of freedom) is rearranged such that this consistency is maintained.

These items are basic features of general coordinate transformations at the level of full quantum gravity, beyond the approximation of semiclassical theory in curved spacetime. In particular, they provide important clues about how complementarity as envisioned in refs. $[15,20]$ may be realized at the microscopic level.

\section{Summary - A grand picture}

The relation between the quantum mechanical view of the world and the spacetime picture of general relativity has never been clear. The issue becomes particularly prominent in a system with a black hole. Quantum mechanics suggests that the black hole formation and evaporation processes are unitary - a black hole appears simply as an intermediate (gigantic) resonance between the initial collapsing matter and final Hawking radiation states. On the other hand, general relativity suggests that a classical observer falling 
into a large black hole does not feel anything special at the horizon. These two, seemingly unrelated, assertions are surprisingly hard to reconcile. With naive applications of standard quantum field theory on curved spacetime, one is led to the conclusion that unitarity of quantum mechanics is violated [9] or that an infalling observer finds something dramatic (firewalls) at the location of the horizon [6-8] (for earlier work, see [42-44]).

In this paper, we have argued that the resolution to this puzzle lies in how a semiclassical description of the system - quantum theory of matter and radiation on a fixed spacetime background - arises from the microscopic theory of quantum gravity. While a semiclassical description employs an exact spacetime background, the quantum uncertainty principle implies that there is no such thing - there is an intrinsic uncertainty for background spacetime for any finite energy and momentum. This implies, in particular, that at the microscopic level there are many different ways to arrive at the same background for the semiclassical theory, within the precision allowed by quantum mechanics. This is the origin of the Bekenstein-Hawking (and related, e.g. Gibbons-Hawking [45]) entropy. The semiclassical picture is obtained after coarse-graining these degrees of freedom representing the microscopic structure of spacetime, which we called the vacuum degrees of freedom. More specifically, any result in semiclassical theory is a statement about the maximally mixed ensemble of microscopic quantum states consistent with the specified background within the required uncertainty [12].

This picture elucidates why the purely semiclassical calculation of ref. [9] finds a violation of unitarity. At the microscopic level, formation and evaporation of a black hole are processes in which information in the initial collapsing matter is converted into that in the vacuum degrees of freedom, which is later transferred back to semiclassical degrees of freedom, i.e. Hawking radiation. Since semiclassical theory is incapable of describing microscopic details of the vacuum degrees of freedom (because it describes them as already coarse-grained, Bekenstein-Hawking entropy), the description of the black hole formation and evaporation processes in semiclassical theory violates unitarity at all stages throughout these processes. This, of course, does not mean that the processes are non-unitary at the fundamental level.

In order to address the unitary evolution and explore its relation with the existence or non-existence of the interior spacetime, we therefore need to discuss the properties of the vacuum degrees of freedom. While the theory governing the detailed microscopic dynamics of these degrees of freedom is not yet fully known, we may include them in our description in the form of a new index - vacuum index - carried by the microscopic quantum states (which we denoted by $k$ and $\kappa$ ) in addition to the indices representing excitations in semiclassical theory and of the stretched horizon. We have argued that these degrees of freedom show peculiar features, which play key roles in addressing the paradoxes discussed in refs. [6-8]:

- Extreme relativenes. In a fixed reference frame, vacuum degrees of freedom may be viewed as distributed (nonlocally) over space. The spacetime distribution of these degrees of freedom, however, changes if we adopt a different reference frame - they are not anchored to spacetime, and rather live in some "holographic space." This 
dependence on the reference frame occurs in a way that the answers to any physical question are consistent with each other when asked in different reference frames. Together with the reference frame dependence of (some of the) semiclassical degrees of freedom, discussed in the earlier literature [5, 46], this comprises basic features of how general coordinate transformations work in the full theory of quantum gravity.

- Spacetime-matter duality. The vacuum degrees of freedom exhibit dual properties of spacetime and matter (even in a description in a single reference frame): while these degrees of freedom are interpreted as representing the way the semiclassical spacetime is realized at the microscopic level, their interactions with semiclassical degrees of freedom make them look like thermal radiation. (At a technical level, the Hilbert space labeled by the vacuum index and that by semiclassical excitations do not factor.) In a sense, these degrees of freedom are neither spacetime nor matter/radiation, as can be seen from the fact that their spacetime distribution changes as we change the reference frame, and that their detailed dynamics cannot be treated in semiclassical theory (as was done in refs. [6-8]). This situation reminds us of wave-particle duality, which played an important role in early days in the development of quantum mechanics - a quantum object exhibited dual properties of waves and particles, while the "true" (quantum) description did not fundamentally rely on either of these classical concepts.

These features make the existence of the black hole interior consistent with unitary evolution, in the sense of complementarity [5] as envisioned in refs. [15, 20]. In particular, a large nearly flat spacetime region near the Schwarzschild horizon becomes manifest in a reference frame whose origin follows a free-fall trajectory starting from rest from a point sufficiently far from the black hole.

It is often assumed that two systems related by the equivalence principle, e.g. a static detector held near the Schwarzschild horizon and an accelerating detector in Minkowski space, must reveal similar physics. This is, however, not true. Since the equivalence principle can make a statement only about a point at a given moment in a given reference frame, while a system in quantum mechanics is specified by a state which generally encodes global information on the equal-time hypersurface, there is no reason that physics of the two systems must be similar beyond a point in space. In particular, a detector reacts very differently to blueshifted Hawking radiation and Unruh radiation in Minkowski space at the microscopic level - it extracts microscopic information about spacetime in the former case, while it does not in the latter.

While our study has focused on a system with a black hole, we do not see any reason why the basic picture we arrived at does not apply to more general cases. We find it enlightening that our results indicate specific properties for the microscopic degrees of freedom that play a crucial role in the emergence of spacetime at the fundamental level. Unraveling the detailed dynamics of these degrees of freedom would be a major step toward obtaining a complete theory of quantum gravity. As a first step, it seems interesting to study implications of our picture for the case that spacetime approaches anti-de Sitter space in the asymptotic region, in which we seem to know a little more [47]. It would also 
be interesting to explore implications of our picture for cosmology, e.g. along the lines of refs. $[15,17,19]$.

\section{Acknowledgments}

We would like to thank Raphael Bousso, Ben Freivogel, Daniel Harlow, Juan Maldacena, Donald Marolf, Joseph Polchinski, Douglas Stanford, Jaime Varela, Erik Verlinde, Herman Verlinde, and I-Sheng Yang for various conversations during our exploration of this subject. Y.N. thanks the Aspen Center for Physics and the National Science Foundation (NSF) Grant \#1066293 for hospitality during his visit in which a part of this work was carried out. F.S. thanks the Department of Energy (DOE) National Nuclear Security Administration Stewardship Science Graduate Fellowship for financial support. This work was supported in part by the Director, Office of Science, Office of High Energy and Nuclear Physics, of the U.S. DOE under Contract DE-AC02-05CH11231, and in part by the NSF under grant PHY-1214644.

Open Access. This article is distributed under the terms of the Creative Commons Attribution License (CC-BY 4.0), which permits any use, distribution and reproduction in any medium, provided the original author(s) and source are credited.

\section{References}

[1] J. Preskill, Do black holes destroy information?, in Blackholes, membranes, wormholes and superstrings, S. Kalara and D.V. Nanopoulos eds., World Scientific, Singapore (1993), hep-th/9209058 [INSPIRE].

[2] G. 't Hooft, The black hole interpretation of string theory, Nucl. Phys. B 335 (1990) 138 [INSPIRE].

[3] C.R. Stephens, G. 't Hooft and B.F. Whiting, Black hole evaporation without information loss, Class. Quant. Grav. 11 (1994) 621 [gr-qc/9310006] [INSPIRE].

[4] W.K. Wootters and W.H. Zurek, A single quantum cannot be cloned, Nature 299 (1982) 802 [INSPIRE].

[5] L. Susskind, L. Thorlacius and J. Uglum, The stretched horizon and black hole complementarity, Phys. Rev. D 48 (1993) 3743 [hep-th/9306069] [INSPIRE].

[6] A. Almheiri, D. Marolf, J. Polchinski and J. Sully, Black holes: complementarity or firewalls?, JHEP 02 (2013) 062 [arXiv:1207.3123] [INSPIRE].

[7] A. Almheiri, D. Marolf, J. Polchinski, D. Stanford and J. Sully, An apologia for firewalls, JHEP 09 (2013) 018 [arXiv: 1304.6483] [INSPIRE].

[8] D. Marolf and J. Polchinski, Gauge/gravity duality and the black hole interior, Phys. Rev. Lett. 111 (2013) 171301 [arXiv: 1307.4706] [INSPIRE].

[9] S.W. Hawking, Breakdown of predictability in gravitational collapse, Phys. Rev. D 14 (1976) 2460 [INSPIRE].

[10] J.D. Bekenstein, Black holes and entropy, Phys. Rev. D 7 (1973) 2333 [INSPIRE]. 
[11] S.W. Hawking, Particle creation by black holes, Commun. Math. Phys. 43 (1975) 199 [Erratum ibid. 46 (1976) 206] [INSPIRE].

[12] Y. Nomura and S.J. Weinberg, Black holes, entropies and semiclassical spacetime in quantum gravity, JHEP 10 (2014) 185 [arXiv:1406.1505] [INSPIRE].

[13] P. Hayden and J. Preskill, Black holes as mirrors: quantum information in random subsystems, JHEP 09 (2007) 120 [arXiv:0708.4025] [INSPIRE].

[14] Y. Sekino and L. Susskind, Fast scramblers, JHEP 10 (2008) 065 [arXiv:0808.2096] [INSPIRE].

[15] Y. Nomura, Quantum mechanics, spacetime locality and gravity, Found. Phys. 43 (2013) 978 [arXiv:1110.4630] [INSPIRE].

[16] Y. Nomura, F. Sanches and S.J. Weinberg, The black hole interior in quantum gravity, arXiv: 1412.7539 [INSPIRE].

[17] Y. Nomura, Physical theories, eternal inflation and quantum universe, JHEP 11 (2011) 063 [arXiv: 1104.2324] [INSPIRE].

[18] B.S. DeWitt, Quantum theory of gravity. 1. The canonical theory, Phys. Rev. 160 (1967) 1113 [INSPIRE].

[19] Y. Nomura, The static quantum multiverse, Phys. Rev. D 86 (2012) 083505 [arXiv: 1205.5550] [INSPIRE].

[20] Y. Nomura, J. Varela and S.J. Weinberg, Low energy description of quantum gravity and complementarity, Phys. Lett. B 733 (2014) 126 [arXiv:1304.0448] [INSPIRE].

[21] D.N. Page, Is black hole evaporation predictable?, Phys. Rev. Lett. 44 (1980) 301 [INSPIRE].

[22] Y. Nomura, J. Varela and S.J. Weinberg, Black holes, information and Hilbert space for quantum gravity, Phys. Rev. D 87 (2013) 084050 [arXiv:1210.6348] [INSPIRE].

[23] G. 't Hooft, Dimensional reduction in quantum gravity, in Salamfestschrift, A. Ali, J. Ellis and S. Randjbar-Daemi eds., World Scientific, Singapore (1994), gr-qc/9310026 [INSPIRE].

[24] Y. Nomura and S.J. Weinberg, Entropy of a vacuum: what does the covariant entropy count?, Phys. Rev. D 90 (2014) 104003 [arXiv:1310.7564] [INSPIRE].

[25] D.N. Page, Average entropy of a subsystem, Phys. Rev. Lett. 71 (1993) 1291 [gr-qc/9305007] [INSPIRE].

[26] Y. Takahashi and H. Umezawa, Thermo field dynamics, Collective Phenomena 2 (1975) 55.

[27] J.B. Hartle and S.W. Hawking, Path integral derivation of black hole radiance, Phys. Rev. D 13 (1976) 2188 [INSPIRE].

[28] W.G. Unruh, Notes on black hole evaporation, Phys. Rev. D 14 (1976) 870 [inSPIRE].

[29] J.M. Bardeen, Black holes do evaporate thermally, Phys. Rev. Lett. 46 (1981) 382 [InSPIRE].

[30] R. Balbinot, Hawking radiation and the back reaction - a first approach, Class. Quant. Grav. 1 (1984) 573.

[31] G. Dvali, Black holes and large- $N$ species solution to the hierarchy problem, Fortsch. Phys. 58 (2010) 528 [arXiv:0706.2050] [INSPIRE].

[32] W.G. Unruh and R.M. Wald, Acceleration radiation and generalized second law of thermodynamics, Phys. Rev. D 25 (1982) 942 [INSPIRE]. 
[33] A.R. Brown, Tensile strength and the mining of black holes, Phys. Rev. Lett. 111 (2013) 211301 [arXiv: 1207.3342] [INSPIRE].

[34] D.N. Page, Information in black hole radiation, Phys. Rev. Lett. 71 (1993) 3743 [hep-th/9306083] [INSPIRE].

[35] D.N. Page, Particle emission rates from a black hole: massless particles from an uncharged, nonrotating hole, Phys. Rev. D 13 (1976) 198 [INSPIRE].

[36] W.H. Zurek, Entropy evaporated by a black hole, Phys. Rev. Lett. 49 (1982) 1683 [INSPIRE].

[37] D.N. Page, Comment on 'entropy evaporated by a black hole', Phys. Rev. Lett. 50 (1983) 1013 [INSPIRE].

[38] L. Susskind, Strings, black holes and Lorentz contraction, Phys. Rev. D 49 (1994) 6606 [hep-th/9308139] [INSPIRE].

[39] W.G. Unruh and R.M. Wald, What happens when an accelerating observer detects a Rindler particle, Phys. Rev. D 29 (1984) 1047 [InSPIRE].

[40] L. Susskind, The world as a hologram, J. Math. Phys. 36 (1995) 6377 [hep-th/9409089] [INSPIRE].

[41] R. Bousso, A covariant entropy conjecture, JHEP 07 (1999) 004 [hep-th/9905177] [INSPIRE].

[42] S.L. Braunstein, S. Pirandola and K. Życzkowski, Better late than never: information retrieval from black holes, Phys. Rev. Lett. 110 (2013) 101301 [arXiv:0907.1190] [INSPIRE].

[43] S.D. Mathur, The information paradox: a pedagogical introduction, Class. Quant. Grav. 26 (2009) 224001 [arXiv:0909.1038] [INSPIRE].

[44] S.B. Giddings, Nonlocality versus complementarity: a conservative approach to the information problem, Class. Quant. Grav. 28 (2011) 025002 [arXiv:0911.3395] [INSPIRE].

[45] G.W. Gibbons and S.W. Hawking, Cosmological event horizons, thermodynamics and particle creation, Phys. Rev. D 15 (1977) 2738 [InSPIRE].

[46] L. Susskind and J. Lindesay, An introduction to black holes, information and the string theory revolution: the holographic universe, World Scientific, Singapore (2005).

[47] J.M. Maldacena, The large- $N$ limit of superconformal field theories and supergravity, Int. J. Theor. Phys. 38 (1999) 1113 [Adv. Theor. Math. Phys. 2 (1998) 231] [hep-th/9711200] [INSPIRE]. 